\title{
RANDOM REGULAR GRAPHS: ASYMPTOTIC DISTRIBUTIONS AND CONTIGUITY
}

\author{
By
}

Svante Janson

IMA Preprint Series \# 1193

November 1993 


\title{
RANDOM REGULAR GRAPHS: ASYMPTOTIC DISTRIBUTIONS AND CONTIGUITY
}

\author{
SVANTE JANSON
}

\begin{abstract}
The asymptotic distribution of the number of Hamilton cycles in a random regular graph is determined. The limit distribution is of an unusual type; it is the distribution of a variable whose logarithm can be written as an infinite linear combination of independent Poisson variables, and thus the logarithm has an infinitely divisible distribution with a certain discrete Lévy measure. Similar results are found for some related problems.

These limit results imply that some different models of random regular graphs are contiguous, which means that they are qualitatively asymptotically equivalent. For example, if $r \geq 3$, then the usual (uniformly distributed) random $r$-regular graph is contiguous to the one constructed by taking the union of $r$ perfect matchings on the same vertex set (assumed to be of even cardinality), conditioned on there being no multiple edges. Some consequences of contiguity for asymptotic distributions are discussed.
\end{abstract}

\section{INTRODUCTION}

In two remarkable papers, Robinson and Wormald [19], [20] have shown that asymptotically almost every random $r$-regular graph contains a Hamilton cycle for every fixed $r \geq 3$. The purpose of this note is to show that their method of proof in fact gives more which is only implicit in their papers.

(We say here and below that "asymptotically almost every" graph has a property, or that a graph property holds "asymptotically almost surely" if the probability tends to 1 as the order of the graph increases; this is often called just "almost every" and "almost surely", but we will in this paper reserve those terms for their usual measure theoretic meaning of probability equal to 1 . Another equivalent term, used by some authors, is "with high probability", whp.)

Robinson and Wormald actually give two different proofs of their result. We will draw further conclusions from both.

In [19] they prove the result for $r=3$ using a conditioning and analysis of variance technique. As we will see below, this proof also gives the asymptotic distribution of the number of Hamilton cycles, which turns out to be rather complicated and neither normal nor log-normal. The method also applies to a number of related situations. We state these results in Section 1, deferring the proofs to Sections 6-7.

In [20], Robinson and Wormald use a different method for $r \geq 4$. It is based on establishing a certain equivalence, known as contiguity, between two different models of random $r$-regular graphs. The two models have different distributions, but nevertheless,

Supported by the Göran Gustafsson Foundation for Research in Natural Sciences and Medicine 
a property which holds asymptotically almost surely for one of the models also holds asymptotically almost surely for the other. (Events having a limiting probability strictly between 0 and 1 need not have the same limiting probability for the other model; contiguity means qualitative but not quantitative equivalence.) We believe that contiguity may be useful also for other problems in combinatorial probability theory, so we study the concept in some detail in Sections 2 and 3, giving both general results and specific examples for random graphs. We show that several models give contiguous distributions. In Section 4 we describe how these results give asymptotic distributions for functionals when we study random regular graphs with one of these distributions instead of the uniform one.

We let in this paper $G(n, r)$ denote a random $r$-regular graph, drawn with uniform distribution from the set of all $r$-regular graphs on a fixed set of $n$ labelled vertices. We assume always that $n$ or $r$ is even, and $n \geq r-1$, otherwise no such graphs exist. (There should be no risk of confusion with the notations $G(n, p)$ and $G(n, m)$ in this paper.) A random $r$-regular graph means always a graph with this, uniform, distribution, unless we explicitly specify another distribution.

We will often use multigraphs and prove versions of our results both for graphs and for multigraphs. One reason for doing this is that several of the proofs become easier in this greater generality.

Acknowledgement. This paper was begun during the conference on Random graphs and combinatorial structures in Oberwolfach in August 1993, and completed during a visit to the Institute for Mathematics and its Applications in Minneapolis later the same year. I thank several of the participants of these meetings, in particular Alan Frieze and Mark Jerrum, for helpful conversations. I am also grateful to Nick Wormald for helpful correspondence.

\section{Asymptotic Distributions.}

As said above, the asymptotic distribution of the number of Hamilton cycles follows from the proof by Robinson and Wormald [19], which is based on conditioning and analysis of variance; see also Cooper, Frieze, Molloy and Reed [8] for comments and a partial reformulation of the argument. We set forth the main steps necessary to derive the asymptotic distribution as a general result.

Theorem 1. Let $\lambda_{i}>0$ and $\delta_{i} \geq-1, i=1,2, \ldots$, be constants and suppose that for each $n$ there are random variables $X_{i n}, i=1,2, \ldots$, and $Y_{n}$ (defined on the same probability space) such that $X_{i n}$ is non-negative integer valued and $\mathrm{E} Y_{n} \neq 0$ (at least for large $n$ ), and furthermore the following conditions are satisfied:

(A1) $X_{i n} \stackrel{d}{\rightarrow} Z_{i}$ as $n \rightarrow \infty$, jointly for all $i$, where $Z_{i} \sim \operatorname{Po}\left(\lambda_{i}\right)$ are independent Poisson random variables;

(A2) For any finite sequence $x_{1}, \ldots, x_{m}$ of non-negative integers,

$$
\frac{\mathrm{E}\left(Y_{n} \mid X_{1 n}=x_{1}, \ldots, X_{m n}=x_{m}\right)}{\mathrm{E} Y_{n}} \rightarrow \prod_{i=1}^{m}\left(1+\delta_{i}\right)^{x_{i}} e^{-\lambda_{i} \delta_{i}} \quad \text { as } n \rightarrow \infty \text {; }
$$

(A3) $\sum_{i} \lambda_{i} \delta_{i}^{2}<\infty$; 
(A4) $\frac{\mathrm{E} Y_{n}^{2}}{\left(\mathrm{E} Y_{n}\right)^{2}} \rightarrow \exp \left(\sum_{i} \lambda_{i} \delta_{i}^{2}\right) \quad$ as $n \rightarrow \infty$.

Then

$$
\frac{Y_{n}}{\mathrm{E} Y_{n}} \stackrel{d}{\rightarrow} W=\prod_{i=1}^{\infty}\left(1+\delta_{i}\right)^{Z_{i}} e^{-\lambda_{i} \delta_{i}} \quad \text { as } n \rightarrow \infty
$$

moreover, this and the convergence in A1 hold jointly. The infinite product defining $W$ converges a.s. and in $L^{2}$, with $\mathrm{E} W=1$ and $\mathrm{E} W^{2}=\exp \left(\sum_{i} \lambda_{i} \delta_{i}^{2}\right)=\lim _{n \rightarrow \infty} \mathrm{E} Y_{n}^{2} /\left(\operatorname{E~} Y_{n}\right)^{2}$. Hence, the normalized variables $Y_{n} / \mathrm{E} Y_{n}$ are uniformly square integrable. Furthermore, the event $W>0$ equals, up to a set of probability zero, the event that $Z_{i}>0$ for some $i$ with $\delta_{i}=-1$. In particular, $W>0$ a.s. if and only if every $\delta_{i}>-1$.

Remarks 1. We define $0^{0}=1$.

2. By joint convergence of an infinite number of variables we mean joint convergence of every finite subset, which is equivalent to convergence in $\mathbf{R}^{\infty}$.

3. We will often omit the "as $n \rightarrow \infty$ " in limits below, when there is no danger of confusion.

4. Condition A4 may be weakened to $\lim _{\sup } \rightarrow \infty \mathrm{E} Y_{n}^{2} /\left(\mathrm{E} Y_{n}\right)^{2} \leq \exp \left(\sum_{i} \lambda_{i} \delta_{i}^{2}\right)$, since it follows from the proof in Section 5 that the other conditions imply $\liminf _{n \rightarrow \infty} \mathrm{E} Y_{n}^{2} /\left(\mathrm{E} Y_{n}\right)^{2}$ $\geq \exp \left(\sum_{i} \lambda_{i} \delta_{i}^{2}\right)$.

5. The values of $\lambda_{i}$ and $\delta_{i}$ are prescribed by A1 and A2. If $\sup _{n} \mathrm{E} X_{i n}^{2}<\infty$ for each $i$, as is the case in all our applications, then $\left\{X_{i n}\right\}_{n}$ and $\left\{X_{i n} Y_{n} / \mathrm{E} Y_{n}\right\}_{n}$ are uniformly integrable (for fixed $i$ ) and we obtain (from the theorem or directly from A1 and A2)

$$
\begin{aligned}
\mathrm{E} X_{i n} & \rightarrow \mathrm{E} Z_{i}=\lambda_{i} \\
\mathrm{E}\left(X_{i n} Y_{n} / \mathrm{E} Y_{n}\right) & \rightarrow \mathrm{E} Z_{i} W=\mathrm{E} Z_{i}\left(1+\delta_{i}\right)^{Z_{i}} e^{-\lambda_{i} \delta_{i}}=\lambda_{i}\left(1+\delta_{i}\right)
\end{aligned}
$$

and thus

$$
\begin{aligned}
\lambda_{i} & =\lim _{n \rightarrow \infty} \mathrm{E} X_{i n} \\
\delta_{i} & =\lim _{n \rightarrow \infty} \mathrm{E}\left(X_{i n} Y_{n}\right) / \mathrm{E} X_{i n} \mathrm{E} Y_{n}-1 .
\end{aligned}
$$

6. The moments of $W$ are easily computed:

$$
\mathrm{E} W^{m}=\exp \left(\sum_{i=1}^{\infty} \lambda_{i}\left(\left(1+\delta_{i}\right)^{m}-1-m \delta_{i}\right)\right), \quad m>0
$$

The sum converges for all $m>0$ if, for example, $\sup \delta_{i}<\infty$. (If moreover inf $\delta_{i}>-1,(1.2)$ holds also for $m<0$.) The conditions in the theorem do not in general imply convergence of higher moments than the second, but we conjecture that in the applications studied in this paper, for every fixed $m>0, \mathrm{E}\left(Y_{n} / \mathrm{E} Y_{n}\right)^{m}$ is bounded and thus converges to the value in (1.2) as $n \rightarrow \infty$. 
7. When $W>0$ a.s., we obtain by (1.1)

$$
\begin{aligned}
\log W & =\sum_{j}\left(\log \left(1+\delta_{j}\right) Z_{j}-\lambda_{j} \delta_{j}\right) \\
& =\sum_{j} \log \left(1+\delta_{j}\right)\left(Z_{j}-\mathrm{E} Z_{j}\right)+\alpha,
\end{aligned}
$$

with $\alpha=\sum_{j} \lambda_{j}\left(\log \left(1+\delta_{j}\right)-\delta_{j}\right)$, and thus $\log W$ has the characteristic function

$$
\mathrm{E} e^{i t \log W}=\exp \left(i t \alpha+\sum_{j} \lambda_{j}\left(e^{i t \log \left(1+\delta_{j}\right)}-1-i t \log \left(1+\delta_{j}\right)\right)\right) .
$$

These equations show that $\log W$ has an infinitely divisible distribution with a discrete Lévy measure, viz. $\sum_{j} \lambda_{j} \delta_{\log \left(1+\delta_{j}\right)}$, cf. e.g. Loève [16].

8. If, for example, $\delta_{i} \rightarrow 0$ as $i \rightarrow \infty$ with $\delta_{i}>-1$, inf $\left|\delta_{i+1} / \delta_{i}\right|>0$ and $\lambda_{i}\left|\delta_{i}\right| \epsilon \rightarrow \infty$ for some $\epsilon>0$, then it is easily shown that the characteristic function of $\log W$ is integrable and thus $\log W$ and $W$ have absolutely continuous distributions, with continuous density functions. This is the case in all applications below, except that sometimes we have a $\delta_{i}=-1$ and then the distribution of $W$ has also a point mass at 0 .

9. It follows from the proof that if we have two or several sequences of variables $Y_{n}^{(j)}$, each satisfying the conditions of the theorem (possibly with different $\delta_{i}^{(j)}$ ) with the same variables $X_{i n}$, then the $Y_{n}^{(j)}$ converge jointly. It follows for example, by the uniform square integrability, that $\mathrm{E} Y_{n}^{(1)} Y_{n}^{(2)} / \mathrm{E} Y_{n}^{(1)} \operatorname{E~} Y_{n}^{(2)} \rightarrow \operatorname{E~} W^{(1)} W^{(2)}=\exp \left(\sum_{i} \lambda_{i} \delta_{i}^{(1)} \delta_{i}^{(2)}\right)$.

10. For convenience, we use the index set $\{1,2, \ldots\}$ in Theorem 1 , but we will without comment replace it by other countable index sets in applications.

We will in all our applications of Theorem 1 verify condition A2 by the method of moments argument used by Robinson and Wormald [19]. We state this step as a separate lemma. We let $(X)_{k}$ denote the descending factorial $X(X-1) \cdots(X-k+1)$.

Lemma 1. Suppose that A1 holds, that $Y_{n} \geq 0$ and that

$$
\mathrm{E}\left(Y_{n}\left(X_{i n}\right)_{j_{1}} \cdots\left(X_{m n}\right)_{j_{m}}\right) / \mathrm{E} Y_{n} \rightarrow \prod_{1}^{m} \mu_{i}^{j_{i}} \quad \text { as } n \rightarrow \infty
$$

for some $\mu_{i} \geq 0$ and every finite sequence $j_{1}, \ldots, j_{m}$ of non-negative integers. Then A2 holds with $\delta_{i}=\mu_{i} / \lambda_{i}-1$.

We will prove Theorem 1 and Lemma 1 in Section 5. The idea of the proof of Theorem 1, following Robinson and Wormald [19] and Cooper, Frieze, Molloy and Reed [8], is to write $Y_{n}=Y_{n}^{\prime}+\left(Y_{n}-Y_{n}^{\prime}\right)$, where $Y_{n}^{\prime}=\mathrm{E}\left(Y_{n} \mid X_{1 n}, X_{2 n}, \ldots\right)$. Then $\operatorname{Var} Y_{n}=\operatorname{Var} Y_{n}^{\prime}+$ $\operatorname{Var}\left(Y_{n}-Y_{n}^{\prime}\right)$ by orthogonality ("analysis of variance"). Let us normalize to $\mathrm{E} Y_{n}=1$. Roughly speaking, the right hand side of A4 then equals the limit of $1+\operatorname{Var} Y_{n}^{\prime}$, and thus A4 says that almost all of the variance of $Y_{n}$ comes from $Y_{n}^{\prime}$, whence $\operatorname{Var}\left(Y_{n}-Y_{n}^{\prime}\right)$ is small. Hence $Y_{n}$ is well approximated by $Y_{n}^{\prime}$, which in turn is well approximated by $W$ by A2 and A1. (The detailed argument in Section 5 involves also a truncation.)

For Hamilton cycles, these general theorems yield the following result. (Proofs of this and the following theorems are given in Section 6.) 
Theorem 2. Let $r \geq 3$ be fixed and let $H_{n}=H(G(n, r))$ be the number of Hamilton cycles in a random $r$-regular graph on $n$ vertices. Then

$$
\frac{H_{n}}{\mathrm{E} H_{n}} \stackrel{d}{\rightarrow} W=\prod_{\substack{i \geq 3 \\ i \text { odd }}}\left(1-\frac{2}{(r-1)^{i}}\right)^{Z_{i}} e^{1 / i}
$$

where $Z_{i} \sim \operatorname{Po}\left(\frac{(r-1)^{i}}{2 i}\right)$ are independent Poisson random variables.

In this case

$$
\mathrm{E} H_{n} \sim e \sqrt{\frac{\pi}{2 n}}\left(\frac{(r-2)^{(r-2) / 2}(r-1)}{r^{(r-2) / 2}}\right)^{n}
$$

and

$$
\mathrm{E} H_{n}^{2} /\left(\mathrm{E} H_{n}\right)^{2} \rightarrow e^{-2 /(r-1)} \frac{r}{r-2}
$$

see $[20]$.

The limiting random variable $W$ in Theorem 2 is positive a.s. by Theorem 1 . Hence the theorem implies

$$
\mathrm{P}\left(H_{n} \geq 1\right)=\mathrm{P}\left(H_{n} / \mathrm{E} H_{n}>0\right) \rightarrow \mathrm{P}(W>0)=1
$$

so our theorem contains the result by Robinson and Wormald.

The limiting distribution in Theorem 2 is more complicated than the ones that usually appear in random graph theory. The Poisson variables $Z_{i}$ that appear have, however, a simple interpretation as the numbers of small cycles in the random regular graph. More precisely, see Theorem 1 and Section 6 , if we let $X_{i n}$ be the number of cycles of length $i$ in the graph, then $X_{i n} \stackrel{d}{\rightarrow} Z_{i}$ as $n \rightarrow \infty$, and this convergence in distribution holds jointly, for all $i$, together with $H_{n} / \mathrm{E} H_{n} \stackrel{d}{\rightarrow} W$. Hence the theorem can be interpreted as saying that $H_{n} / \mathrm{E} H_{n}$ is, with large probability, well approximated by the infinite product with $Z_{i}$ replaced by the small cycle count $X_{i n}$, or rather by a finite product $\prod_{1}^{N}(1-$ $\left.2 /(r-1)^{2 k+1}\right)^{X_{2 k+1, n}} e^{1 /(2 k+1)}$. (We have to let $n \rightarrow \infty$ first and then $N \rightarrow \infty$ in order to get convergence, because the factors $e^{1 /(2 k+1)}$, which are necessary convergence factors in (1.3), act as divergence factors for any finite $n$.)

It should perhaps not be surprising that $H_{n}$ thus essentially is determined by the numbers of small cycles. Similar results hold for $G(n, p)$ and $G(n, m)$, where for certain ranges of $p$ and $m, \log H_{n}$ is well approximated by a linear function of the number of edges, and of the number of paths of length 2, respectively, see [12]. Those results are simpler, however, since $\log H_{n}$ then is asymptotically normal, while in the present case $\log H_{n}-\log \mathrm{E} H_{n}$ converges to $\log W$ which has a rather complicated infinitely divisible distribution. Moreover, for $G(n, p)$ and $G(n, m)$ it suffices to use one small subgraph count in the approximation, whereas we here need an infinite sequence. (There are parallels to this in $G(n, p)$ for other functionals, see e.g. [1], [11].)

Note that every small subgraph count for regular graphs can be essentially expressed in terms of small cycle counts, since asymptotically almost every random regular graph has 
no small multicyclic subgraphs. This explains why only cycle counts appear in Theorem 2 (as $Z_{i}$ ), but it seems mysterious that only the odd cycle counts appear, while the even cycle counts are asymptotically independent of $H_{n}$.

Theorem 1 applies also to several other, related, problems and we give some examples.

First, recall that random regular graphs are simplest generated by the configuration models of Bender and Canfield [3] or Bollobás [4], [5], which can be described as follows. Given $r$ and a vertex set $V$ with $n$ elements (with $r n$ even), define a configuration to be a perfect matching of the $r n$ elements in $V \times[r]$, where $[r]=\{1, \ldots, r\}$. (We can call these $r n$ elements half-edges.) Every configuration projects to an $r$-regular multigraph on $V$, and we define a random $r$-regular multigraph to be the projection of a randomly (uniformly) chosen configuration. It is easily seen that if we condition on there being no loops or multiple edges, we obtain a random $r$-regular graph $G(n, r)$ with the usual uniform distribution. Note, however, that our random regular multigraphs are not uniformly distributed; the probability of obtaining a specified multigraph has a weight factor of $1 / 2$ for every loop and $1 / k$ ! for every set of $k$ parallel edges. Nevertheless, this model of a random regular multigraph seems to be the simplest and most useful.

We use $G^{*}(n, r)$ to denote a random regular multigraph (with this distribution). We let $X_{i n}$ be the number of cycles of length $i \geq 1$ in our random multigraph, and observe that the multigraph is a graph if and only if $X_{1 n}=X_{2 n}=0$. It is well-known that, as $n \rightarrow \infty$, $X_{i n} \stackrel{d}{\rightarrow} Z_{i} \sim \operatorname{Po}\left(\frac{1}{2 i}(r-1)^{i}\right)$ jointly for $i \geq 1$, with $Z_{i}$ independent, and thus,

$$
\mathrm{P}\left(X_{1 n}=X_{2 n}=0\right) \rightarrow \mathrm{P}\left(Z_{1}=Z_{2}=0\right)=\exp \left(-\frac{1}{2}(r-1)-\frac{1}{4}(r-1)^{2}\right)>0 ;
$$

see $[5]$.

We may now extend Theorem 2 to multigraphs. In this and the following theorems, just as in Theorem 2 , the variables $Z_{i}$ are the limits of the cycle counts $X_{i n}$.

Theorem 3. Let $r \geq 3$ be fixed and let $H_{n}^{*}=H\left(G^{*}(n, r)\right)$ be the number of Hamilton cycles in a random $r$-regular multigraph on $n$ vertices. Then

$$
\frac{H_{n}^{*}}{\mathrm{E} H_{n}^{*}} \stackrel{d}{\rightarrow} W=\prod_{\substack{i \geq 1 \\ i \text { odd }}}\left(1-\frac{2}{(r-1)^{i}}\right)^{Z_{i}} e^{1 / i}
$$

where $Z_{i} \sim \operatorname{Po}\left(\frac{(r-1)^{i}}{2 i}\right)$ are independent Poisson random variables.

For multigraphs we have, cf. (1.4) and (1.5)

$$
\mathrm{E} H_{n}^{*} \sim \sqrt{\frac{\pi}{2 n}}\left(\frac{(r-2)^{(r-2) / 2}(r-1)}{r^{(r-2) / 2}}\right)^{n}
$$

and

$$
\mathrm{E}\left(H_{n}^{*}\right)^{2} /\left(\mathrm{E} H_{n}^{*}\right)^{2} \rightarrow \frac{r}{r-2}
$$

see Frieze, Jerrum and Molloy [9]. 
Note that the limit distribution for multigraphs in Theorem 3 differs from the one for graphs in Theorem 2 only by the additional factor $\left(1-\frac{2}{r-1}\right)^{Z_{1}}$ e, where $Z_{1} \sim \operatorname{Po}\left(\frac{r-1}{2}\right)$ should be interpreted as (the limit of) the number of 1-cycles, i.e. loops, in the multigraph.

If $r \geq 4$, so that $1-\frac{2}{r-1}>0$, then the limit $W>0$ a.s. also in Theorem 3 , and thus the theorem implies that asymptotically almost every $r$-regular multigraph contains a Hamilton cycle. On the other hand, if $r=3$, then $1-\frac{2}{r-1}=0$, and thus $W=0$ when $Z_{1}>0$ but, as before, $W>0$ a.s. when $Z_{1}=0$.

It follows from the joint convergence of $\left(H_{n}^{*}, X_{1 n}\right)$ to $\left(W, Z_{1}\right)$ that asymptotically almost every cubic multigraph is Hamiltonian if and only if it lacks loops, in the sense that as $n \rightarrow \infty$, the probability tends to 0 that a random cubic multigraph has one of these properties but not the other. (One implication is obvious for all cubic multigraphs; the other holds only asymptotically.)

Furthermore, the joint convergence of $\left(H_{n}^{*}, X_{1 n}, X_{2 n}\right)$ and the fact that $\mathrm{P}\left(Z_{1}=Z_{2}=\right.$ $0)>0$ imply that the limit in Theorem 3 remains true if we condition on $X_{1 n}=X_{2 n}=0$, i.e.

$$
\mathcal{L}\left(\frac{H_{n}^{*}}{\mathrm{E} H_{n}^{*}} \mid X_{1 n}=X_{2 n}=0\right) \stackrel{d}{\rightarrow} \mathcal{L}\left(W \mid Z_{1}=Z_{2}=0\right)=\mathcal{L}\left(e \prod_{\substack{i \geq 3 \\ i \text { odd }}}\left(1-\frac{2}{(r-1)^{i}}\right)^{Z_{i}} e^{1 / i}\right) .
$$

The left hand side is the distribution of the number $H_{n}=H(G(n, r))$ of Hamilton cycles in a random regular graph, normalized by the expectation $\mathrm{E} H_{n}^{*}$ for the random multigraph. Since the uniform integrability of $H_{n}^{*} / \mathrm{E} H_{n}^{*}$ (see Theorem 1) survives the conditioning,

$$
\mathrm{E} H_{n} / \mathrm{E} H_{n}^{*}=\mathrm{E}\left(H_{n}^{*} \mid X_{1 n}=X_{2 n}=0\right) / \mathrm{E} H_{n}^{*} \rightarrow \mathrm{E}\left(W \mid Z_{1}=Z_{2}=0\right)=e,
$$

and we recover Theorem 1 as a corollary to Theorem 3 .

For future reference we observe that we can use this conditioning argument in all cases where Theorem 1 applies. We obtain, if $Y_{n}^{\prime}$ has the distribution of $Y_{n}$ conditioned on $X_{1 n}=X_{2 n}=0$,

$$
\mathrm{E} Y_{n}^{\prime} / \mathrm{E} Y_{n} \rightarrow \mathrm{E}\left(W \mid Z_{1}=Z_{2}=0\right)=\exp \left(-\lambda_{1} \delta_{1}-\lambda_{2} \delta_{2}\right)
$$

and similarly

$$
\mathrm{E}\left(Y_{n}^{\prime}\right)^{2} /\left(\mathrm{E} Y_{n}\right)^{2} \rightarrow \mathrm{E}\left(W^{2} \mid Z_{1}=Z_{2}=0\right)=\exp \left(-2 \lambda_{1} \delta_{1}-2 \lambda_{2} \delta_{2}+\sum_{i=3}^{\infty} \lambda_{i} \delta_{i}^{2}\right)
$$

combining these we obtain

$$
\mathrm{E}\left(Y_{n}^{\prime}\right)^{2} /\left(\mathrm{E} Y_{n}^{\prime}\right)^{2} \rightarrow \exp \left(\sum_{i=3}^{\infty} \lambda_{i} \delta_{i}^{2}\right)=\exp \left(-\lambda_{1} \delta_{1}^{2}-\lambda_{2} \delta_{2}^{2}\right) \mathrm{E} W^{2}
$$

For the Hamilton cycles in Theorem 3 we have $\delta_{1}=-2 /(r-1), \delta_{2}=0$ and $\lambda_{1}=(r-1) / 2$, and we see that (1.9) and (1.11) yield (1.4) and (1.5) from (1.7) and (1.8).

As said above, the proof of Theorem 2 is based on the proof that $\mathrm{P}\left(H_{n}>0\right) \rightarrow 1$ for $r=3$ by Robinson and Wormald [19]. In [20] they prove the result for $r \geq 4$ by another method, which does not give the information on the distribution that we need. One step in that proof, however, is to use the method of [19] to prove a result on the number $M$ of perfect matchings. Again, their proof contains implicitly the asymptotic distribution. 
Theorem 4. Let $M_{n}^{*}=M\left(G^{*}(n, r)\right)$ be the number of perfect matchings in a random $r$-regular multigraph. Then, if $r \geq 3$ is fixed and $n$ is even,

$$
\frac{M_{n}^{*}}{\mathrm{E} M_{n}^{*}} \stackrel{d}{\rightarrow} W=\prod_{i=1}^{\infty}\left(1+\frac{(-1)^{i}}{(r-1)^{i}}\right)^{Z_{i}} e^{(-1)^{i-1} / 2 i}
$$

where $Z_{i} \sim \operatorname{Po}\left(\frac{(r-1)^{i}}{2 i}\right)$ are as in Theorems 1 and 3. Similarly, for graphs, with $M_{n}=$ $M(G(n, r))$,

$$
\frac{M_{n}}{\mathrm{E} M_{n}} \stackrel{d}{\rightarrow} \prod_{i=3}^{\infty}\left(1+\frac{(-1)^{i}}{(r-1)^{i}}\right)^{Z_{i}} e^{(-1)^{i-1 / 2 i}}
$$

For matchings,

$$
\mathrm{E} M_{n}^{*} \sim \sqrt{2}\left(\frac{(r-1)^{(r-1) / 2}}{r^{(r-2) / 2}}\right)^{n}
$$

and

$$
\mathrm{E}\left(M_{n}^{*}\right)^{2} /\left(\mathrm{E} M_{n}^{*}\right)^{2} \rightarrow \sqrt{\frac{r-1}{r-2}}
$$

while (1.9) and (1.11) yield $\mathrm{E} M_{n} \sim e^{1 / 4} \mathrm{E} M_{n}^{*}$ and

$$
\mathrm{E} M_{n}^{2} /\left(\mathrm{E} M_{n}\right)^{2} \rightarrow e^{-(2 r-1) / 4(r-1)^{2}} \mathrm{E}\left(M_{n}^{*}\right)^{2} /\left(\mathrm{E} M_{n}^{*}\right)^{2}
$$

cf. [6] and [20].

As for Theorems 1 and $3, Z_{i}$ can be interpreted (asymptotically) as the number of cycles of length $i$. Note that now cycles of all lengths appear, and that the number of perfect matchings is positively correlated with the even cycle counts but negatively correlated with the odd ones.

In Theorem 4, every $\delta_{i}>-1$, and thus $W>0$ a.s. and we obtain as a corollary the well-known result that asymptotically almost every $r$-regular (multi-)graph of even order contains a perfect matching when $r \geq 3$.

Remark 9 implies that $H_{n}^{*}$ and $M_{n}^{*}$ converge jointly to the limits in Theorems 3 and 4; moreover,

$$
\mathrm{E} H_{n}^{*} M_{n}^{*} / \mathrm{E} H_{n}^{*} \mathrm{E} M_{n}^{*} \rightarrow \exp \left(\sum_{i \text { odd }} \lambda_{i} \frac{-2}{(r-1)^{i}} \frac{-1}{(r-1)^{i}}\right)=\sqrt{\frac{r}{r-2}} .
$$

Instead of studying just single perfect matchings in an $r$-regular graph, we may try to decompose it into an $r$-tuple of perfect matchings. For any $r$-regular multigraph $G^{*}$, let $U\left(G^{*}\right)$ be the number of partitions of the edge set of $G^{*}$ into $r$ perfect matchings $M_{1}, \ldots, M_{r}$. To be specific, we distinguish between different orderings of $M_{1}, \ldots, M_{r}$, and we regard parallel edges in $G^{*}$ as distinguishable. We may also say that $U\left(G^{*}\right)$ is the number of $r$-colourings of the edges in $G^{*}$ such that each colour class is a perfect matching. We believe that the following result holds for any $r \geq 3$, but we have only been able to prove it for $r=3$. 
Theorem 5. Let $U_{n}^{*}=U\left(G^{*}(n, r)\right)$ and $U_{n}=U(G(n, r))$. If $r=3$, then (for even $n$ )

$$
\frac{U_{n}^{*}}{\mathrm{E} U_{n}^{*}} \stackrel{d}{\rightarrow} \prod_{i=1}^{\infty}\left(1-\frac{1}{(r-1)^{i-1}}\right)^{Z_{i}} e^{(r-1) / 2 i}
$$

and

$$
\frac{U_{n}}{\mathrm{E} U_{n}} \stackrel{d}{\rightarrow} \prod_{i=3}^{\infty}\left(1-\frac{1}{(r-1)^{i-1}}\right)^{Z_{i}} e^{(r-1) / 2 i}
$$

where $Z_{i} \sim \operatorname{Po}\left(\frac{(r-1)^{i}}{2 i}\right)$ are as above.

The asymptotics of the expectations and variances are given in Section 6. Note that in this case, $\delta_{1}=-(r-1)^{0}=-1$ while $\delta_{i}>-1$ for $i \geq 2$; hence we obtain as a corollary that asymptotically almost every cubic multigraph without loops has a decomposition into perfect matchings. (Of course, a loop always prevents such a decomposition.) This is true for any $r \geq 3$, as was proved (for graphs) by Robinson and Wormald [19], [20]; we will prove a stronger statement in Theorem 11 below. See also Molloy, Robalewska-Szarłat, Robinson and Wormald [17].

There are also analogous results for some related random structures.

Let us first consider a random regular bipartite graph $B(n, r)$ (on $n+n$ vertices) or the corresponding random multigraph $B^{*}(n, r)$. As for $G(n, r)$, these are obtained from random configurations; the allowed configurations now are perfect matchings between two disjoint sets $V_{1} \times[r]$ and $V_{2} \times[r]$ of cardinality $r n$. In this case, for the small cycle counts,

$$
X_{i n} \stackrel{d}{\rightarrow} Z_{i} \sim \operatorname{Po}\left(\frac{(r-1)^{i}}{i}\right) \quad \text { as } n \rightarrow \infty,
$$

for even $i\left(i \geq 2\right.$ for $B^{*}(n, r)$ and $i \geq 4$ for $\left.B(n, r)\right)$; of course, $X_{i n}=0$ when $i$ is odd. For perfect matchings, there is an analogue to Theorem 4 .

Theorem 6. Let $M_{n}^{*}=M\left(B^{*}(n, r)\right)$. If $r \geq 3$, then

$$
\frac{M_{n}^{*}}{\mathrm{E} M_{n}^{*}} \stackrel{d}{\rightarrow} \prod_{\substack{i \geq 2 \\ i \text { even }}}\left(1+\frac{1}{(r-1)^{i}}\right)^{Z_{i}} e^{-1 / i} .
$$

For $M(B(n, r))$, we get the same result with $i \geq 4$ in the product.

We have

$$
\mathrm{E} M_{n}^{*} \sim\left(\frac{2 \pi(r-1) n}{r}\right)^{1 / 2}\left(\frac{(r-1)^{r-1}}{r^{r-2}}\right)^{n}
$$

and

$$
\mathrm{E}\left(M_{n}^{*}\right)^{2} /\left(\mathrm{E} M_{n}^{*}\right)^{2} \rightarrow \frac{r-1}{\sqrt{r(r-2)}}
$$

the corresponding results for $B(n, r)$ follow by (1.9) and (1.11), cf. Bollobas and McKay $[6]$. 
For Hamilton cycles in the bipartite case, it has been shown by Robinson and Wormald [18] that for $r=3$, with $H_{n}=H(B(n, r))$, E $H_{n}^{2} /\left(\mathrm{E} H_{n}\right)^{2} \rightarrow 1$ and thus $H_{n} / \mathrm{E} H_{n} \stackrel{p}{\rightarrow} 1$. We believe that the same holds for any $r>3$ and also for $B^{*}(n, r), r \geq 3$, but as far as we know this has not yet been verified. Note that this result, although strikingly different from Theorem 1, still fits nicely together with it, since the variation in Theorem 1 comes from the randomness in the small odd cycle counts, whereas there are no odd cycles in a bipartite graph. (That asymptotically almost every $r$-regular bipartite graph contains a Hamilton cycle when $r \geq 3$ was proved by Robinson and Wormald [20].)

The theory also applies to the random $r$-regular digraph $D(n, r)$ (also known as $r$-in, $r$-out), and the corresponding directed multigraph $D^{*}(n, r)$. We can construct these by a configuration model with $2 r$ half-edges for each vertex, $r$ labeled "in" and $r$ labeled "out", or, equivalently, by taking a random bipartite (multi-)graph with vertex set $V_{1} \cup V_{2}$, orienting all edges from $V_{1}$ to $V_{2}$ and then identifying $V_{1}$ and $V_{2}$ (by a fixed identification).

For directed graphs, we again use the numbers of small cycles in the limit theorems, but we now consider cycles of any possible orientation. If $C$ is a $k$-cycle with oriented edges, let $k_{i}, i=0,1,2$, be the numbers of vertices with out-degree $i$ in $C$. Clearly, $k_{2}=k_{0}$ and $k_{0}+k_{1}+k_{2}=k$. Let $X_{C n}$ be the number of copies of $C$ in $D^{*}(n, r)$. Then, by standard arguments,

$$
\mathrm{E} X_{C n}=\frac{(n)_{k}(r(r-1))^{k_{0}+k_{2}} r^{2 k_{1}}}{\operatorname{aut}(C)(r n)_{k}} \rightarrow \lambda_{C}=\frac{1}{\operatorname{aut}(C)} r^{k_{1}}(r-1)^{k_{0}+k_{2}},
$$

where $\operatorname{aut}(C)$ is the number of automorphisms of $C$, and

$$
X_{C n} \stackrel{d}{\rightarrow} Z_{C} \sim \operatorname{Po}\left(\lambda_{C}\right),
$$

jointly in all $C$, with $Z_{C}$ independent.

With some foresight, we introduce special notation for two classes of oriented cycles, viz. $C_{k}, k \geq 1$, for the directed cycle of length $k$ and $C_{k}^{a}, k \geq 2$ even, for the cycle of length $k$ with alternating directions. (These are the cycles with all and no vertices of out-degree 1 , respectively.) By (1.19) we have

$$
\lambda_{C_{k}}=\frac{r^{k}}{k}
$$

and, for even $k$,

$$
\lambda_{C_{k}^{a}}=\frac{(r-1)^{k}}{k} .
$$

For simplicity, we state the results for random directed multigraphs only. The corresponding results for directed graphs follow by conditioning on $Z_{C_{2}^{a}}=0$, or $Z_{C_{1}}=Z_{C_{2}^{a}}=0$, or $Z_{C_{1}}=Z_{C_{2}}=Z_{C_{2}^{a}}=0$, depending on what we regard as a directed graph; we leave the details to the reader.

Theorem 7. Let $H_{n}=H\left(D^{*}(n, r)\right)$ be the number of Hamilton cycles in a random $r$-regular directed multigraph. If $r \geq 3$, then

$$
\frac{H_{n}}{\mathrm{E} H_{n}} \stackrel{d}{\rightarrow} \prod_{k=1}^{\infty}\left(1-\frac{1}{r^{k}}\right)^{Z_{C_{k}}} e^{1 / k} \prod_{\substack{j=2 \\ j \text { even }}}^{\infty}\left(1+\frac{1}{(r-1)^{j}}\right)^{Z_{C_{j}^{a}}} e^{-1 / j}
$$


where $Z_{C} \sim \operatorname{Po}\left(\lambda_{C}\right)$ are independent.

We here have

$$
\mathrm{E} H_{n} \sim \sqrt{\frac{2 \pi(r-1)}{r n}}\left(\frac{(r-1)^{r-1}}{r^{r-2}}\right)^{n}
$$

and

$$
\mathrm{E} H_{n}^{2} /\left(\mathrm{E} H_{n}\right)^{2} \rightarrow \sqrt{\frac{r}{r-2}}
$$

Again, the limit in the theorem is $>0$ a.s., and thus we obtain as a corollary the result by Cooper, Frieze and Molloy [7] that asymptotically almost every $r$-regular directed (multi)graph is Hamiltonian, $r \geq 3$.

Another interesting quantity in a directed graph is the number of permutations, or 1regular subgraphs. Note that the correspondence between bipartite graphs and directed graphs induces a correspondence between perfect matchings and permutations; moreover, cycles in the bipartite graph correspond to alternating cycles in the directed graph, except in the improbable cases where vertices coincide.

Hence the following theorem is just a reformulation of Theorem 6 .

Theorem 8. Let $V_{n}=V\left(D^{*}(n, r)\right)$ be the number of permutations in a random $r$-regular directed multigraph. If $r \geq 3$, then

$$
\frac{V_{n}}{\mathrm{E} V_{n}} \stackrel{d}{\rightarrow} \prod_{\substack{j \geq 2 \\ j \text { even }}}\left(1+\frac{1}{(r-1)^{j}}\right)^{Z_{C_{j}^{a}}} e^{-1 / j}
$$

where $Z_{C_{j}^{a}} \sim \operatorname{Po}\left(\lambda_{C_{j}^{a}}\right)$ are as above.

The expectation and second moment are given by (1.17) and (1.18).

Finally, we give an application to random regular hypergraphs. Cooper, Frieze, Molloy and Reed [8] have generalized the proof in [20] on existence of perfect matchings to regular hypergraphs. Again, their proof yields the asymptotic distribution. We refer to [8] for definitions.

Theorem 9. Let $s \geq 3$ and $r$ be fixed, with $r$ so large that $\log r /(r-1) \log \frac{r}{r-1}+1>s$. If $Y$ is the number of perfect matchings in a random $r$-regular $s$-uniform hypergraph, then

$$
\frac{Y}{\operatorname{EY}} \stackrel{d}{\rightarrow} W=\prod_{i=2}^{\infty}\left(1+\frac{(-1)^{i}}{(r-1)^{i}}\right)^{Z_{i}} e^{-(1-s)^{i} / 2 i}
$$

where $Z_{i} \sim \operatorname{Po}\left(\frac{1}{2 i}((r-1)(s-1))^{i}\right)$ are independent.

\section{Contiguity in General}

Definition. Let $\left(P_{n}\right)_{1}^{\infty}$ and $\left(Q_{n}\right)_{1}^{\infty}$ be two sequences of probability measures, such that for each $n, P_{n}$ and $Q_{n}$ both are defined on the same measurable space $\left(\Omega_{n}, \mathcal{F}_{n}\right)$. We then say that the sequences are contiguous if for every sequence of measurable sets $A_{n} \subset \Omega_{n}$,

$$
\lim _{n \rightarrow \infty} P_{n}\left(A_{n}\right)=0 \Longleftrightarrow \lim _{n \rightarrow \infty} Q_{n}\left(A_{n}\right)=0 .
$$


Clearly, contiguity is an equivalence relation among sequences of probability measures on a given sequence of space $\left(\Omega_{n}\right)$. Its use is mainly in statistics, but it seems to be natural and useful also in the study of random combinatorial structures. In that case, typically, $\Omega_{n}$ is a (finite) set of some combinatorial objects of size $n, \mathcal{F}_{n}$ is the $\sigma$-field of all subsets of $\Omega_{n}$, and $P_{n}$ and $Q_{n}$ are probability measures corresponding to two different ways of selecting an element of $\Omega_{n}$ "at random".

This notion was introduced by Le Cam [13], see also Le Cam [14], [15] and Roussas [21]. We will here consider only the symmetric version of contiguity defined above, although the corresponding one-sided notion have been studied as well, see [15].

There are many equivalent definitions of contiguity; some are given below and several others are given in the references given above. For completeness we will include proofs even when our results can be found in these references.

It is important to realize that contiguity is an asymptotic property of two sequences of probability measures; if we say that two probability measures (or distributions, or random models) are contiguous, we really mean that there is some parameter $n$ (although perhaps not explicitly mentioned) that tends to infinity. If we, as is often useful, informally regard asymptotic results as statements about a fictitious infinite limiting model, then contiguity can be interpreted as mutual absolute continuity of the two probability measures. (In this context it may be observed that in the special case that $\Omega_{n}, \mathcal{F}_{n}, P_{n}$ and $Q_{n}$ do not depend on $n$, contiguity reduces to mutual absolute continuity.)

Note also that the definition says nothing about the rates of convergence of $P_{n}\left(A_{n}\right)$ and $Q_{n}\left(A_{n}\right)$; these may be quite different. It is, nevertheless, possible to restate the definition in terms of estimates, which, however, use unknown functions to relate the rates. (In certain examples, it may of course be possible to replace these by explicit functions.) We given two such reformulations, which we think may be useful for a better understanding the contiguity concept.

Proposition 1. The sequences $\left(P_{n}\right)$ and $\left(Q_{n}\right)$ are contiguous if and only if there exist a sequence $\varepsilon_{n} \rightarrow 0$ and a continuous function $\varphi:[0,1] \rightarrow[0,1]$ with $\varphi(0)=0$ such that for every $n$ and $A_{n} \in \mathcal{F}_{n}$

$$
P_{n}\left(A_{n}\right) \leq \varepsilon_{n}+\varphi\left(Q_{n}\left(A_{n}\right)\right) \quad \text { and } \quad Q_{n}\left(A_{n}\right) \leq \varepsilon_{n}+\varphi\left(P_{n}\left(A_{n}\right)\right) .
$$

Proof. If $\left(P_{n}\right)$ and $\left(Q_{n}\right)$ are contiguous, then for every $\varepsilon>0$ there exist $n(\varepsilon)$ and $\delta(\varepsilon)$ such that $n \geq n(\varepsilon)$ and $P_{n}\left(A_{n}\right) \leq \delta(\varepsilon) \Rightarrow Q_{n}\left(A_{n}\right) \leq \varepsilon$, and similarly with $P_{n}$ and $Q_{n}$ interchanged. The existence of $\left(\varepsilon_{n}\right)$ and $\varphi$ now follows easily. The converse is obvious.

For the next results, we recall that each $Q_{n}$ has a Lebesgue decomposition $Q_{n}=Q_{n}^{a}+Q_{n}^{s}$ where $Q_{n}^{a}$ is absolutely continuous with respect to $P_{n}$ while $Q_{n}^{s}$ and $P_{n}$ are mutually singular, and that there exists a function $d Q_{n} / d P_{n} \geq 0$ (the Radon-Nikodym derivative) such that

$$
Q_{n}^{a}\left(A_{n}\right)=\int_{A_{n}} \frac{d Q_{n}}{d P_{n}} d P_{n}, \quad A_{n} \in \mathcal{F}_{n}
$$

(If $\Omega_{n}$ is countable, the measures are given by probability functions $p_{n}(w)$ and $q_{n}(w)$; in this case $Q_{n}^{s}$ is the restriction of $Q_{n}$ to the set $\left\{w: p_{n}(w)=0\right\}$, while $d Q_{n} / d P_{n}=q_{n}(w) / p_{n}(w)$ when $p_{n}(w) \neq 0$ (and arbitrary otherwise).) 
Proposition 2. The sequences $\left(P_{n}\right)$ and $\left(Q_{n}\right)$ are contiguous if and only if for every $\varepsilon>0$ there exist $n(\varepsilon)$ and $K(\varepsilon)$ such that for every $n \geq n(\varepsilon)$ there exists a set $B_{n} \in \mathcal{F}_{n}$ with $P_{n}\left(B_{n}^{c}\right) \leq \varepsilon, Q_{n}\left(B_{n}^{c}\right) \leq \varepsilon$ such that

$$
K(\varepsilon)^{-1} \leq \frac{Q_{n}\left(A_{n}\right)}{P_{n}\left(A_{n}\right)} \leq K(\varepsilon) \quad \text { for all } A_{n} \subseteq B_{n}
$$

Remark 11. We may here replace (2.3) by the equivalent condition that the restriction of $Q_{n}$ to $B_{n}$ is absolutely continuous with respect to $P_{n}$, with

$$
K(\varepsilon)^{-1} \leq \frac{d Q_{n}}{d P_{n}} \leq K(\varepsilon) \quad P_{n} \text {-a.s. on } B_{n} .
$$

Proof. Suppose that $\left(P_{n}\right)$ and $\left(Q_{n}\right)$ are contiguous. Fix a large number $K$, let $C_{n}$ be a set such that $P_{n}\left(C_{n}\right)=0=Q_{n}^{s}\left(C_{n}^{c}\right)$ (this is possible by the definition of singular measures), and define

$$
D_{n}=\left\{w \in \Omega_{n}: d Q_{n} / d P_{n}>K\right\} .
$$

Then

$$
P_{n}\left(C_{n} \cup D_{n}\right)=\int_{D_{n}} d P_{n} \leq \frac{1}{K} \int_{D_{n}} \frac{d Q_{n}}{d P_{n}} d P_{n}=\frac{1}{K} Q_{n}^{a}\left(D_{n}\right) \leq \frac{1}{K} .
$$

Hence Proposition 1 yields $Q_{n}\left(C_{n} \cup D_{n}\right) \leq \varepsilon_{n}+\varphi\left(K^{-1}\right)$. Furthermore, for any $A_{n} \subseteq$ $\left(C_{n} \cup D_{n}\right)^{c}$,

$$
Q_{n}\left(A_{n}\right)=Q_{n}^{a}\left(A_{n}\right)=\int_{A_{n}} \frac{d Q_{n}}{d P_{n}} d P_{n} \leq K P_{n}\left(A_{n}\right)
$$

We similarly define $C_{n}^{\prime}$ and $D_{n}^{\prime}$ with the roles of $P_{n}$ and $Q_{n}$ interchanged, and let $B_{n}=$ $\left(C_{n} \cup D_{n} \cup C_{n}^{\prime} \cup D_{n}^{\prime}\right)^{c}$. Then $P_{n}\left(B_{n}^{c}\right), Q_{n}\left(B_{n}^{c}\right) \leq K^{-1}+\varepsilon_{n}+\varphi\left(K^{-1}\right)$, which is less than $\varepsilon$ for $n \geq n(\varepsilon)$ if $n(\varepsilon)$ and $K=K(\varepsilon)$ are large enough.

Conversely, if such sets $B_{n}$ exist, then for large $n$ and every $A_{n} \in \mathcal{F}_{n}$,

$$
Q_{n}\left(A_{n}\right) \leq Q_{n}\left(A_{n} \cap B_{n}\right)+Q_{n}\left(B_{n}^{c}\right) \leq K(\varepsilon) P_{n}\left(A_{n}\right)+\varepsilon .
$$

In particular, if $P_{n}\left(A_{n}\right) \rightarrow 0$, then $\lim \sup Q_{n}\left(A_{n}\right) \leq \varepsilon$, for every $\varepsilon>0$, and thus $Q_{n}\left(A_{n}\right) \rightarrow$ 0 . By symmetry, we obtain also the converse implication and thus the sequences are contiguous.

For the next result, we consider for simplicity only the case when $d Q_{n} / d P_{n}$ converges in distribution.

Proposition 3. Suppose that $L_{n}=d Q_{n} / d P_{n}$, regarded as a random variable on $\left(\Omega_{n}, \mathcal{F}_{n}\right.$, $\left.P_{n}\right)$, converges in distribution to some random variable $L$ as $n \rightarrow \infty$. Then $\left(P_{n}\right)$ and $\left(Q_{n}\right)$ are contiguous if and only if $L>0$ a.s. and $\mathrm{E} L=1$.

Proof. Suppose that $\left(P_{n}\right)$ and $\left(Q_{n}\right)$ are contiguous. Let $\varepsilon>0$, and let $K(\varepsilon)$ and $B_{n}$, $n \geq n(\varepsilon)$, be as in Proposition 2. In particular, by Remark $11, K(\varepsilon)^{-1} \leq L_{n} \leq K(\varepsilon)$ on $B_{n}$. Thus

$$
\mathrm{P}(L=0) \leq \mathrm{P}\left(L<K(\varepsilon)^{-1}\right) \leq \limsup _{n \rightarrow \infty} P_{n}\left(L_{n}<K(\varepsilon)^{-1}\right) \leq \limsup _{n \rightarrow \infty} P_{n}\left(B_{n}^{c}\right) \leq \varepsilon .
$$


Moreover, since $L_{n} \wedge K(\varepsilon) \stackrel{d}{\rightarrow} L \wedge K(\varepsilon)$ as $n \rightarrow \infty$, dominated convergence yields

$$
\begin{aligned}
\mathrm{E} L & \geq \mathrm{E}(L \wedge K(\varepsilon))=\lim _{n \rightarrow \infty} \int_{\Omega_{n}} L_{n} \wedge K(\varepsilon) d P_{n} \geq \limsup _{n \rightarrow \infty} \int_{B_{n}} L_{n} d P_{n}=\limsup _{n \rightarrow \infty} Q_{n}\left(B_{n}\right) \\
& \geq 1-\varepsilon .
\end{aligned}
$$

Since $\varepsilon$ is arbitrary, $\mathrm{P}(L=0)=0$ and $\mathrm{E} L=1$.

Conversely, suppose that $\mathrm{P}(L=0)=0$ and $\mathrm{E} L=1$, and let $\varepsilon>0$. There exists $\delta>0$ such that $\mathrm{P}(L \leq \delta)<\varepsilon$; we may furthermore choose $\delta$ such that $\delta<1$ and $\mathrm{P}(L=\delta)=0$. Then $P_{n}\left(L_{n} \leq \delta\right) \rightarrow \mathrm{P}(L \leq \delta)$, and thus $P_{n}\left(L_{n} \leq \delta\right)<\varepsilon$ for large $n$, which also yields $Q_{n}^{a}\left(L_{n} \leq \delta\right)=\int_{L_{n} \leq \delta} L_{n} d P_{n}<\varepsilon \delta \leq \varepsilon$.

We can also find $K>1 / \varepsilon$ such that $\mathrm{E}(L \wedge K)>1-\varepsilon$ and thus $\lim _{n \rightarrow \infty} \int\left(L_{n} \wedge K\right) d P_{n}=$ $\mathrm{E}(L \wedge K)>1-\varepsilon$. Hence, for large $n$, say $n \geq n(\varepsilon)$,

$$
\begin{aligned}
\varepsilon & >1-\int_{\Omega_{n}}\left(L_{n} \wedge K\right) d P_{n}=Q_{n}^{s}\left(\Omega_{n}\right)+Q_{n}^{a}\left(\Omega_{n}\right)-\int_{\Omega_{n}}\left(L_{n} \wedge K\right) d P_{n} \\
& =Q_{n}^{s}\left(\Omega_{n}\right)+\int_{\Omega_{n}}\left(L_{n}-L_{n} \wedge K\right) d P_{n} \geq Q_{n}^{s}\left(\Omega_{n}\right)+\frac{1}{2} \int_{L_{n}>2 K} L_{n} d P_{n} \\
& =Q_{n}^{s}\left(\Omega_{n}\right)+\frac{1}{2} Q_{n}^{a}\left(L_{n}>2 K\right) .
\end{aligned}
$$

Let $N_{n}$ be a set with $P_{n}\left(N_{n}\right)=Q_{n}^{s}\left(N_{n}^{c}\right)=0$, and define $B_{n}=\left\{w \in N_{n}^{c}: \delta \leq L_{n} \leq 2 K\right\}$. Then, for $n \geq n(\varepsilon)$,

$$
P_{n}\left(B_{n}^{c}\right)=P_{n}\left(L_{n}<\delta\right)+P_{n}\left(L_{n}>2 K\right)<\varepsilon+\int L_{n} d P_{n} / 2 K<2 \varepsilon
$$

and

$$
Q_{n}\left(B_{n}^{c}\right)=Q_{n}^{s}\left(\Omega_{n}\right)+Q_{n}^{a}\left(L_{n}<\delta\right)+Q_{n}^{a}\left(L_{n}>2 K\right)<3 \varepsilon .
$$

Hence Proposition 2 implies that $\left(P_{n}\right)$ and $\left(Q_{n}\right)$ are contiguous.

Contiguity is preserved by some natural operations, as the next proposition shows. In (ii) and (iii) we suppose that $\left(\Omega_{n}^{\prime}, \mathcal{F}_{n}^{\prime}\right)$ is another sequence of measure spaces.

Proposition 4. Suppose that $\left(P_{n}\right)$ and $\left(Q_{n}\right)$ are contiguous.

(i) If $\left(A_{n}\right)$ is any sequence of events such that liminf $P_{n}\left(A_{n}\right)>0$, then the conditioned measures $P_{n}\left(\cdot \mid A_{n}\right)$ and $Q_{n}\left(\cdot \mid A_{n}\right)$ are contiguous.

(ii) If $f_{n}: \Omega_{n} \rightarrow \Omega_{n}^{\prime}$ are measurable functions, then the induced measures $P_{n} \circ f_{n}^{-1}$ and $Q_{n} \circ f_{n}^{-1}$ on $\Omega_{n}^{\prime}$ are contiguous.

(iii) If $P_{n}^{\prime}$ and $Q_{n}^{\prime}$ are contiguous probability measures on $\Omega_{n}^{\prime}$, then the product measures $P_{n} \times P_{n}^{\prime}$ and $Q_{n} \times Q_{n}^{\prime}$ on $Q_{n} \times Q_{n}^{\prime}$ are contiguous.

Proof. (i) and (ii) follow easily from the definition (note that also liminf $Q_{n}\left(A_{n}\right)>0$ in (i)); for (iii) it is perhaps simplest to use Proposition 2 with Remark 11. We omit the details. 
Contiguity of two sequences $\left(P_{n}\right)$ and $\left(Q_{n}\right)$ has several useful consequences for limit theorems. The definition says that any property which holds under $P_{n}$ with probability tending to 1 as $n \rightarrow \infty$ (i.e., asymptotically almost surely) also holds under $Q_{n}$ with probability tending to 1 (and conversely). An immediate consequence is that if $X_{n}$ are any random variables such that $X_{n}$ under $P_{n}$ converges in probability to some constant $c$, then $X_{n}$ converges in probability to the same constant $c$ also under $Q_{n}$.

For convergence in distribution we need some further information. In the three next results we suppose that $S$ and $S^{\prime}$ are two complete separable metric spaces, for example $\mathbf{R}, \mathbf{R}^{d}$ or the space $\mathbf{R}^{\infty}$ of infinite sequences. (See [2] for details on convergence in metric spaces.) We use $\mathcal{L}(X \mid P)$ to denote the distribution of $X$ under $P$.

In the first result we assume joint convergence under $P_{n}$ of the variables $X_{n}$ and the Radon-Nikodym derivatives $d Q_{n} / d P_{n}$.

Proposition 5. Suppose that $\left(P_{n}\right)$ and $\left(Q_{n}\right)$ are contiguous, and let $L_{n}=d Q_{n} / d P_{n}$. Suppose further that $X_{n}$ are random variables defined on $\Omega_{n}$ with values in $S$, such that $\mathcal{L}\left(\left(X_{n}, L_{n}\right) \mid P_{n}\right) \rightarrow \mathcal{L}(X, L)$ for some random variables $X$ and $L$ (with values in $S$ and $\mathbf{R}$ respectively), defined on a probability space $(\Omega, \mathcal{F}, P)$. Then $\mathcal{L}\left(X_{n} \mid Q_{n}\right) \rightarrow \mathcal{L}(X \mid Q)$, where $d Q=L d P$ (i.e., $Q(A)=\int_{A} L d P$ ).

Proof. Let $f$ be a bounded continuous real-valued function on $S$. Then $\mathcal{L}\left(f\left(X_{n}\right) L_{n} \mid\right.$ $\left.P_{n}\right) \rightarrow \mathcal{L}(f(X) L)$. If $f \geq 0$, then Fatou's lemma yields

$$
\liminf \int f\left(X_{n}\right) L_{n} d P_{n} \geq \int f(X) L d P .
$$

Since $\int L_{n} d P_{n} \rightarrow \int L d P$ by Proposition 3 , we see, by adding a constant to $f$, that (2.5) actually holds for every $f$. But then we may also substitute $-f$ in (2.5), and obtain

$$
\int f\left(X_{n}\right) d Q_{n}^{a}=\int f\left(X_{n}\right) L_{n} d P_{n} \rightarrow \int f(X) L d P=\int f(X) d Q .
$$

Since also $Q_{n}^{s}\left(\Omega_{n}\right) \rightarrow 0$, which for example follows from (2.6) with $f=1$, we obtain

$$
\int f\left(X_{n}\right) d Q_{n} \rightarrow \int f(X) d Q
$$

for every such $f$, which gives the result.

Proposition 6. Suppose that $\left(P_{n}\right)$ and $\left(Q_{n}\right)$ are contiguous. Suppose further that $X_{n}$ and $Y_{n}$ are random variables defined on $\Omega_{n}$, with values in $S$ and $S^{\prime}$ respectively, such that

$$
\mathcal{L}\left(\left(X_{n}, Y_{n}\right) \mid P_{n}\right) \rightarrow \mathcal{L}(X, f(X))
$$

for some random variable $X$ with values in $S$ and some measurable function $f: S \rightarrow S^{\prime}$. If, furthermore, $\mathcal{L}\left(X_{n} \mid Q_{n}\right) \rightarrow \mathcal{L}\left(X^{\prime}\right)$ for some other random variable $X^{\prime}$, then $\mathcal{L}\left(\left(X_{n}, Y_{n}\right) \mid\right.$ $\left.Q_{n}\right) \rightarrow \mathcal{L}\left(X^{\prime}, f\left(X^{\prime}\right)\right)$; in particular $\mathcal{L}\left(Y_{n} \mid Q_{n}\right) \rightarrow \mathcal{L}\left(f\left(X^{\prime}\right)\right)$.

Proof. Let $L_{n}=d Q_{n} / d P_{n}$. The assumption implies by Prohorov's theorem, see [2], that $\left\{\mathcal{L}\left(\left(X_{n}, Y_{n}\right) \mid P_{n}\right)\right\}$ is a tight family of distributions on $S \times S^{\prime}$. Since also the family 
$\left\{\mathcal{L}\left(L_{n} \mid P_{n}\right)\right\}$ is tight, because $\int L_{n} d P_{n} \leq 1$, the family $\left\{\mathcal{L}\left(\left(X_{n}, Y_{n}, L_{n}\right) \mid P_{n}\right)\right\}$ is tight on $S \times S^{\prime} \times R$. By Prohorov's theorem again, there exists a subsequence that converges in distribution to some variable $(\widetilde{X}, \widetilde{Y}, L)$. Obviously $\mathcal{L}(\tilde{X}, \tilde{Y})=\mathcal{L}(X, f(X))$, and thus $\widetilde{Y}=f(\widetilde{X})$ a.s. Moreover, we may use Proposition 5 on $\left(X_{n}, Y_{n}\right)$ along the subsequence and conclude

$$
\mathcal{L}\left(\left(X_{n}, Y_{n}\right) \mid Q_{n}\right) \rightarrow \mathcal{L}((\widetilde{X}, \tilde{Y}) \mid Q)=\mathcal{L}((\tilde{X}, f(\widetilde{X})) \mid Q)
$$

Since also, by assumption, $\mathcal{L}\left(X_{n} \mid Q_{n}\right) \rightarrow \mathcal{L}\left(X^{\prime}\right)$, we have $\mathcal{L}(\widetilde{X} \mid Q)=\mathcal{L}\left(X^{\prime}\right)$ and thus $\mathcal{L}((\tilde{X}, f(\tilde{X})) \mid Q)=\mathcal{L}\left(X^{\prime}, f\left(X^{\prime}\right)\right)$. Consequently, $\mathcal{L}\left(\left(X_{n}, Y_{n}\right) \mid Q_{n}\right) \rightarrow \mathcal{L}\left(X^{\prime}, f\left(X^{\prime}\right)\right)$ along the subsequence.

We have proved that the sought conclusion holds at least along a subsequence. But the same applies to every subsequence of the original sequence, which thus has a subsubsequence for which $\mathcal{L}\left(\left(X_{n}, Y_{n}\right) \mid Q_{n}\right) \rightarrow \mathcal{L}\left(X^{\prime}, f\left(X^{\prime}\right)\right)$. This implies that the full sequence converges.

The next result gives a necessary condition for contiguity.

Proposition 7. Suppose that $\left(P_{n}\right)$ and $\left(Q_{n}\right)$ are contiguous, and that $X_{n}$ are random variables defined on $\Omega_{n}$ with values in $S$. Suppose further that $\left(X_{n}\right)$ converges in distribution under both $\left(P_{n}\right)$ and $\left(Q_{n}\right)$ :

$$
\mathcal{L}\left(X_{n} \mid P_{n}\right) \rightarrow P_{X} \quad \text { and } \quad \mathcal{L}\left(X_{n} \mid Q_{n}\right) \rightarrow Q_{X}
$$

for two probability measures $P_{X}$ and $Q_{X}$ on $S$. Then $P_{X}$ and $Q_{X}$ are mutually absolutely continuous.

Proof. Let $L_{n}=d Q_{n} / d P_{n}$. Since $\mathrm{E} L_{n} \leq 1$, the sequence $\left(L_{n}\right)$ is tight and we may, by restricting attention to a subsequence, assume that $\mathcal{L}\left(\left(X_{n}, L_{n}\right) \mid P_{n}\right)$ converges. Proposition 5 now implies that $Q_{X}$ is absolutely continuous with respect to $P_{X}$. The converse holds by symmetry.

We will use Proposition 7 in conjunction with the following standard result. (In fact, the given conditions are equivalent to mutual absolute continuity.)

Proposition 8. Suppose that the measure $\prod_{i} \operatorname{Po}\left(\lambda_{i}\right)$ and $\prod_{i} \operatorname{Po}\left(\lambda_{i}^{\prime}\right)$ on $\mathbf{Z}^{\infty}$ are mutually absolutely continuous. Then

$$
\lambda_{i}=0 \Longleftrightarrow \lambda_{i}^{\prime}=0,
$$

and

$$
\sum_{i} \frac{\left(\lambda_{i}-\lambda_{i}^{\prime}\right)^{2}}{\lambda_{i}+\lambda_{i}^{\prime}}<\infty
$$

Proof. The first conclusion is trivial. For the second, we may use the Hellinger integral, defined for any two probability measures $\mu$ and $\mu^{\prime}$ on the same space by $I\left(\mu, \mu^{\prime}\right)=$ 
$\int\left(\frac{d \mu}{d \nu} \frac{d \mu^{\prime}}{d \nu}\right)^{1 / 2} d \nu$, where $\nu$ is any $\sigma$-finite measure with $\mu, \mu^{\prime} \ll \nu$. It is easily seen that $\mu$ and $\mu^{\prime}$ are mutually singular if and only if $I\left(\mu, \mu^{\prime}\right)=0$, and that in our case

$$
I\left(\prod_{i} \operatorname{Po}\left(\lambda_{i}\right), \prod_{i} \operatorname{Po}\left(\lambda_{i}^{\prime}\right)\right)=\prod_{i} I\left(\operatorname{Po}\left(\lambda_{i}\right), \operatorname{Po}\left(\lambda_{i}^{\prime}\right)\right)=\prod_{i} e^{-\left(\sqrt{\lambda_{i}}-\sqrt{\lambda_{i}^{\prime}}\right)^{2} / 2} .
$$

Hence the measures are mutually singular unless

$$
\infty>\sum\left(\sqrt{\lambda_{i}}-\sqrt{\lambda_{i}^{\prime}}\right)^{2}=\sum\left(\lambda_{i}-\lambda_{i}^{\prime}\right)^{2} /\left(\sqrt{\lambda_{i}}+\sqrt{\lambda_{i}^{\prime}}\right)^{2} \geq \frac{1}{2} \sum\left(\lambda_{i}-\lambda_{i}^{\prime}\right)^{2} /\left(\lambda_{i}+\lambda_{i}^{\prime}\right) .
$$

\section{CONTIGUITY FOR REGULAR GRAPHS}

The results of Sections 1 and 2 may now be combined to yield various examples of contiguity. To begin with, let us again consider the number $H_{n}^{*}$ of Hamilton cycles in a random $r$-regular multigraph. Let $\Omega_{n}$ be the set of all $r$-regular multigraphs on the set $V=[n]$, let the probability measure $P_{n}$ on $\Omega_{n}$ be the distribution of the random multigraph $G^{*}(n, r)$, and define a new probability measure $Q_{n}$ on $\Omega_{n}$ by $d Q_{n} / d P_{n}=H_{n}^{*} / \mathrm{E} H_{n}^{*}$, i.e., $Q_{n}(A)=\mathrm{E}\left(H_{n}^{*} 1_{A}\right) / \mathrm{E} H_{n}^{*}$ for any set $A \subseteq Q_{n}$, where $1_{A}$ is the indicator function. Then Theorem 3 and Proposition 3 imply that if $r \geq 4$ then $\left(P_{n}\right)$ and $\left(Q_{n}\right)$ are contiguous. (Recall that we need $r \geq 4$ in order to have $W>0$ a.s. in Theorem 3 and that $\mathrm{E} W=1$ by Theorem 1.)

The measure $Q_{n}$ has the following interpretation. Consider the set $\bar{\Omega}_{n}$ of all pairs $(\widetilde{G}, \widetilde{H}$ ) of a configuration $\widetilde{G}$ (on $n r$ half-edges) and a set $\widetilde{H}$ of edges in $\widetilde{G}$ that projects to a Hamilton cycle in the multigraph $G^{*}$ obtained by projecting $\widetilde{G}$. Pick one of these pairs $(\widetilde{G}, \widetilde{H})$ at random, uniformly over $\bar{\Omega}_{n}$, and take the projection $G^{*}$. This defines a random $r$-regular multigraph which has the distribution $Q_{n}$, since the probability of obtaining a specific multigraph $G^{*}$ is proportional to the number of pairs $(\widetilde{G}, \widetilde{H}) \in \bar{\Omega}$ projecting to $G^{*}$, which is proportional to $P_{n}\left(G^{*}\right) H\left(G^{*}\right)$. Furthermore, by symmetry, we obtain the same distribution $Q_{n}$ by picking the pair $(\widetilde{G}, \widetilde{H})$ at random in the subset $\bar{\Omega}_{n}^{\prime}$ of all such pairs $(\widetilde{G}, \widetilde{H})$ such that the edges in $\widetilde{H}$ join half-edges in $V \times\{1,2\}$. But these pairs are just the pairs $\left(\widetilde{H} \cup \widetilde{H}^{\prime}, \widetilde{H}\right)$, where $\widetilde{H}$ is a configuration on $V \times\{1,2\}$ projecting to a Hamilton cycle and $\widetilde{H}^{\prime}$ is any configuration on $V \times\{3, \ldots, r\}$. Picking an element in $\bar{\Omega}^{\prime}$ at random is thus the same as picking such $\widetilde{H}$ and $\widetilde{H}^{\prime}$ independently at random, and after projecting we see that $Q_{n}$ equals the distribution of the union of a random Hamilton cycle and a random $(r-2)$-regular multigraph $G^{*}(n, r-2)$.

Summing up, and introducing the notations $\approx$ for contiguity, + for the union of two independent random multigraphs on the same vertex set, and $H(n)$ for a random Hamilton cycle on $n$ vertices,

$$
G^{*}(n, r-2)+H(n) \approx G^{*}(n, r), \quad r \geq 4 .
$$

Similarly we obtain from Theorem 4 and Proposition 3, recalling that a random perfect matching is the same as $G^{*}(n, 1)=G(n, 1)$ and assuming $n$ even,

$$
G^{*}(n, r-1)+G(n, 1) \approx G^{*}(n, r), \quad r \geq 3 .
$$


(This is essentially proved in [20], but our use of multigraphs seems to simplify both the result and the proof.)

We note also that the operation + is commutative and associative, and that it behaves well under contiguity in the following sense.

Lemma 2. Suppose that $G_{n}, H_{n}, G_{n}^{\prime}, H_{n}^{\prime}$ are random multigraphs on $n$ vertices with some distributions such that $G_{n} \approx G_{n}^{\prime}$ and $H_{n} \approx H_{n}^{\prime}$. Then $G_{n}+H_{n} \approx G_{n}^{\prime}+H_{n}^{\prime}$.

Proof. By Proposition 4(iii), the pairs $\left(G_{n}, H_{n}\right)$ and $\left(G_{n}^{\prime}, H_{n}^{\prime}\right)$ are contiguous, and the result follows by Proposition 4(ii).

The contiguity (3.1) does not hold for $r=3$ for the simple reason that neither $G^{*}(n, 1)$ nor $H(n)$ can contain any loop, and thus $\mathrm{P}\left(G^{*}(n, 1)+H(n)\right.$ is loopless $)=1$, while $\mathrm{P}\left(G^{*}(n, 3)\right.$ is loopless $)=\mathrm{P}\left(X_{1 n}^{*}=0\right) \rightarrow e^{-\lambda_{1}}=e^{-1}$. Let us therefore define the random $r$-regular loopless multigraph $G^{\prime}(n, r)$ to be $G^{*}(n, r)$ conditioned on $X_{1 n}=0$ (i.e. no loops). This is thus intermediate between $G^{*}(n, r)$ and $G(n, r)$. (As for $G^{*}(n, r)$, the distribution is not the uniform one: there is a weight factor $k !^{-1}$ for every edge with multiplicity $k$.) By conditioning on $X_{1 n}=0$ in Theorem 3, arguing as in Section 1, we see that analogues of Theorems 2,3 and 4 hold for $G^{\prime}(n, r)$ with $W>0$ a.s. for every $r \geq 3$. We can now argue as above and obtain

$$
G^{\prime}(n, r-2)+H(n) \approx G^{\prime}(n, r), \quad r \geq 3
$$

and (for even $n$ )

$$
G^{\prime}(n, r-1)+G(n, 1) \approx G^{\prime}(n, r), \quad r \geq 3 .
$$

Remark 12. In particular,

$$
G^{\prime}(n, 2)+G^{\prime}(n, 1) \approx G^{\prime}(n, 3) \approx H(n)+G^{\prime}(n, 1)
$$

although $G^{\prime}(n, 2) \not \approx H(n)$. This shows that cancellation is not allowed and that there is no unique subtraction corresponding to + .

We can extend (3.3) and (3.4).

Theorem 10. If $r, s \geq 1$, and $(r, s) \neq(1,1)$ or $(2,2)$, then, for even $n$,

$$
G^{\prime}(n, r)+G^{\prime}(n, s) \approx G^{\prime}(n, r+s) .
$$

For any $r \geq 1$ we also have

$$
G^{\prime}(n, r)+H(n) \approx G^{\prime}(n, r+2) .
$$

Proof. The contiguity (3.6) is just a restatement of (3.3). For (3.5), we may assume $r \geq s$. The case $s=1$ is (3.4). We may thus assume $r \geq s \geq 2$, and thus $r \geq 3$ (because $r=s=2$ is excluded). If $r=3$, then (3.3) and (3.4) yield, using Lemma 2,

$$
G^{\prime}(n, 3)+G^{\prime}(n, s) \approx G^{\prime}(n, 1)+H(n)+G^{\prime}(n, s) \approx G^{\prime}(n, 1)+G^{\prime}(n, s+2) \approx G^{\prime}(n, s+3)
$$


while for larger $r$ we use induction in $r$ and (3.4), yielding

$G^{\prime}(n, r)+G^{\prime}(n, s) \approx G^{\prime}(n, r-1)+G^{\prime}(n, 1)+G^{\prime}(n, s) \approx G^{\prime}(n, r-1)+G^{\prime}(n, s+1) \approx G^{\prime}(n, r+s)$.

The contiguity (3.5) is certainly false for $r=s=1$, since $G(n, 1)+G(n, 1)$ has no odd cycles, but the case $r=s=2$, which is left open by our proof, is presumably not really an exception.

Conjecture 1.

$$
G^{\prime}(n, 2)+G^{\prime}(n, 2) \approx G^{\prime}(n, 4) .
$$

We also conjecture a stronger version of Robinson and Wormald [20, Conjecture 1]. Conjecture 2.

$$
H(n)+H(n) \approx G^{\prime}(n, 4) .
$$

Note that the mixed sum is no problem: $G^{\prime}(n, 2)+H(n) \approx G(n, 4)$ by $(3.3)$.

We conjecture also that (3.5) holds for odd $n$ as well, provided $r$ and $s$ are even. (This would follow from Conjecture 2 and (3.3) by an easy induction.)

We now turn to Theorem 5. Again we condition on there being no loops, and obtain by the argument above

$$
G^{\prime}(n, 3) \cong G(n, 1)+G(n, 1)+G(n, 1) .
$$

Using (3.4) and induction, this can be extended to sums of any number (except 2) of perfect matchings. This result has also been obtained (independently) by Molloy, RobalewskaSzarłat, Robinson and Wormald [17].

Theorem 11. Let $r \neq 2$. Then, for even $n$,

$$
G^{\prime}(n, r) \approx G(n, 1)+\cdots+G(n, 1) \quad(r \text { terms }) .
$$

It follows easily from Theorems 10 and 11 that, at least for even $n$, any sum of 3 or more independent $G^{\prime}\left(n, r_{i}\right)$ is contiguous to $G^{\prime}\left(n, \sum r_{i}\right)$, except possibly when every $r_{i}=2$. We may here also replace some terms $G^{\prime}(n, 2)$ by $H(n)$, and the only possible exceptions are when all terms equal $G^{\prime}(n, 2)$ or when all equal $H(n)$.

We have worked with the loopless multigraphs $G^{\prime}(n, r)$ which were easy to handle. We conjecture that similar results hold when we allow loops.

Conjecture 3. If $r, s \geq 1$ and $(r, s) \neq(1,1)$, then

$$
G^{*}(n, r)+G^{*}(n, s) \approx G^{*}(n, r+s) .
$$

Results for random regular graphs follow from the results above by conditioning on there being no multiple edges, cf. Proposition 4(i). Note, however, that the union of two graphs in general is a multigraph, so the results say, for example, that $G(n, r)$ is contiguous 
to the union of $r$ independent perfect matchings, conditioned on there being no multiple edges.

For random regular bipartite and directed graphs, we obtain some similar (but less complete) results from Theorems 6-8; we leave the details to the reader. A bipartite version of Theorem 11 has recently been proved by Hania Robalewska-Szarłat (personal communication). Note, moreover, that if the variance for Hamilton cycles in a random bipartite multigraph is as expected,

$$
\mathrm{E} H\left(B^{*}(n, r)\right)^{2} /\left(\mathrm{E} H\left(B^{*}(n, r)\right)\right)^{2} \rightarrow 1 \quad \text { for } r \geq 3
$$

then not only $B^{*}(n, r) \approx B(n, r-2)+H(2 n)$ would follow; these two models actually would be asymptotically equivalent in the stronger sense that $\lim _{n \rightarrow \infty} P_{n}\left(A_{n}\right)=\lim _{n \rightarrow \infty} Q_{n}\left(A_{n}\right)$ for any events $A_{n}$ such that one of the limits exists.

For $r=2$, we have here considered the models $G^{*}(n, 2), H(n)$ and $G(n, 1)+G(n, 1)$ (and conditioned versions of them). A fourth interesting model is $D^{0}(n, 1)$, obtained by ignoring the directions of the edges in the random digraph $D(n, 1)$, which is the same as a random permutation. (In this model, the probability of a certain 2-regular multigraph is proportional to $2^{\# \text { cycles }}$.) These four models are all non-contiguous, even if we condition on no loops and no multiple edges; this is easily seen by Proposition 7 and 8, letting $X_{n} \in \mathbf{Z}^{\infty}$ be the vector $\left(X_{i n}\right)_{i=3}^{\infty}$, since we have, for the means of the limiting Poisson variables,

$$
\lambda_{i}= \begin{cases}1 / 2 i & \text { for } G^{*}(n, 2) \\ 0 & \text { for } H(n) \\ \left(1+(-1)^{i}\right) / 2 i & \text { for } G(n, 1)+G(n, 1) \\ 1 / i & \text { for } D^{0}(n, 1) .\end{cases}
$$

Nevertheless, we have seen that adding an independent $G(n, 1)$ to one of the three first models (and conditioning on no multiple edges) yields three contiguous distributions; we conjecture that adding it to the fourth also gives a contiguous distribution. Similarly, we conjecture that the sum of any two of these four (different or not) is contiguous to $G(n, 4)$ after conditioning on being simple.

Let us finally prove another contiguity result. Recall that we have defined the random multigraph $G^{*}(n, r)$ as obtained from a uniformly distributed configuration. The distribution of $G^{*}(n, r)$ is not uniform on the set of all $r$-regular multigraphs on $n$ given vertices (unless $r=1$ ), but it is contiguous to the uniform distribution.

Theorem 12. For any fixed $r \geq 1, G^{*}(n, r)$ is contiguous to a random $r$-regular multigraph chosen with the uniform distribution.

Proof. Let $P_{n}$ be the distribution of $G^{*}(n, r)$ and let $Q_{n}$ be the uniform distribution on the set of all $r$-regular multigraphs on $[n]$.

Define, for a given multigraph, $Y_{n}^{\prime}=2^{X_{1 n}}$ and $Y_{n}^{\prime \prime}=\prod_{j=2}^{r}(j !)^{m_{j}}$, where $X_{1 n}$ as usual is the number of loops and $m_{j}$ is the number of edges of multiplicity $j$. Let $Y_{n}=Y_{n}^{\prime} Y_{n}^{\prime \prime}$. It is easily seen that $d P_{n} / d Q_{n}=c_{n} Y_{n}^{-1}$, or $d Q_{n} / d P_{n}=c_{n}^{-1} Y_{n}$, where $c_{n}$ is a normalizing constant; in fact, $c_{n}=\mathrm{E}_{P_{n}} Y_{n}$. The probability that $G^{*}(n, r)$ has any triple edges tends 
to 0 as $n \rightarrow \infty$, and thus $P_{n}\left(Y_{n}^{\prime \prime} \neq 2^{X_{2 n}}\right) \rightarrow 0$. Consequently, under $P_{n}$,

$$
Y_{n} \stackrel{d}{\rightarrow} Y=2^{Z_{1}+Z_{2}},
$$

where $Z_{i} \sim \operatorname{Po}\left((r-1)^{i} / 2 i\right)$ are as usual.

We will show that the variables $Y_{n}$ are uniformly integrable under $P_{n}$. This implies

$$
c_{n}=\mathrm{E}_{P_{n}} Y_{n} \rightarrow \mathrm{E} Y<\infty,
$$

and thus, under $P_{n}$,

$$
\frac{d Q_{n}}{d P_{n}} \stackrel{d}{\rightarrow}(\mathrm{E} Y)^{-1} Y
$$

The result then follows by Proposition 3, because $Y \geq 1$ and thus $(\mathrm{E} Y)^{-1} Y>0$.

To show that $Y_{n}$ are uniformly integrable, it suffices to show that $\sup _{n} \mathrm{E} Y_{n}^{2}<\infty$ (all expectations in the remainder of the proof are for $P_{n}$ ). By Hölder's inequality it suffices to prove $\sup _{n} \mathrm{E}\left(Y_{n}^{\prime}\right)^{4}<\infty$ and $\sup _{n} \mathrm{E}\left(Y_{n}^{\prime \prime}\right)^{4}<\infty$. Now $\left(Y_{n}^{\prime}\right)^{4}=2^{4 X_{1 n}}$. Define $\widetilde{X}_{n}=\sum_{j=2}^{r} m_{j}$, the number of multiple edges (counted just once each). Then, crudely, $Y_{n}^{\prime \prime} \leq(r !)^{\widetilde{X}_{n}}$ and thus $\left(Y_{n}^{\prime \prime}\right)^{4} \leq\left(r !^{4}\right)^{\widetilde{X}_{n}}$.

Let $p_{k}$ be the probability that a random configuration contains $k$ given edges, $1 \leq k \leq$ $r n / 2$. Then $p_{k}=\prod_{i=1}^{k}(r n-2 i+1)^{-1}$, and thus, using Stirling's formula,

$$
p_{k}^{1 / k} \leq p_{r n / 2}^{2 / r n}=\left(\frac{2^{r n / 2}(r n / 2) !}{(r n) !}\right)^{2 / r n} \leq \frac{e}{r n} .
$$

There are $\left(\begin{array}{l}r \\ 2\end{array}\right) n \leq r^{2} n$ possible edges in the configuration that will create loops. Hence

$$
\mathrm{P}\left(X_{1 n}=k\right) \leq\left(\begin{array}{c}
r^{2} n \\
k
\end{array}\right) p_{k} \leq \frac{1}{k !}\left(r^{2} n\right)^{k}\left(\frac{e}{r n}\right)^{k}=\frac{1}{k !}(e r)^{k}
$$

and

$$
\mathrm{E} 2^{4 X_{1 n}} \leq \sum_{k=0}^{\infty} \frac{1}{k !} 2^{4 k}(e r)^{k}=\exp (16 e r)<\infty .
$$

Similarly, there are less than $n^{2} r^{4}$ pairs of edges in the configuration that will create parallel edges. Hence

$$
\mathrm{P}\left(\widetilde{X}_{n}=k\right) \leq \frac{1}{k !}\left(n^{2} r^{4}\right)^{k} p_{2 k} \leq \frac{1}{k !}\left(e^{2} r^{2}\right)^{k}
$$

and

$$
\mathrm{E}\left(Y_{n}^{\prime \prime}\right)^{4} \leq \mathrm{E}\left(r !^{4}\right)^{\widetilde{X}_{n}} \leq \exp \left(e^{2} r^{2} r !^{4}\right)<\infty .
$$

This completes the proof.

Remark 13. By (3.10) and (3.9), under $P_{n}$,

$$
\frac{d Q_{n}}{d P_{n}} \stackrel{d}{\rightarrow}(\mathrm{E} Y)^{-1} Y=e^{-\lambda_{1}-\lambda_{2}} 2^{Z_{1}+Z_{2}}
$$


and this convergence holds jointly with $X_{i n} \stackrel{d}{\rightarrow} Z_{i}$. Proposition 5 applied to a vector of cycle counts $\left(X_{i n}\right)_{i=1}^{m}$, with $m \geq 2$ fixed, yields, with $\lambda_{i}=(r-1)^{i} / 2 i$,

$$
\begin{aligned}
Q_{n}\left(X_{1 n}=x_{1}, \ldots, X_{m n}=x_{m}\right) & \rightarrow Q_{n}\left(Z_{1}=x_{1}, \ldots, Z_{m}=x_{m}\right) \\
& =e^{-\lambda_{1}-\lambda_{2}} 2^{x_{1}+x_{2}} \mathrm{P}\left(Z_{1}=x_{1}, \ldots, Z_{m}=x_{m}\right) \\
& =\prod_{i=1}^{2} \frac{\left(2 \lambda_{i}\right)^{x_{i}}}{x_{i} !} e^{-2 \lambda_{i}} \prod_{i=3}^{m} \frac{\lambda_{i}^{x_{i}}}{x_{i} !} e^{-\lambda_{i}} .
\end{aligned}
$$

Hence, for a random $r$-regular multigraph with the uniform distribution, $X_{i n} \stackrel{d}{\rightarrow} \operatorname{Po}\left(\lambda_{i}^{\prime}\right)$, with independent limits, where

$$
\lambda_{i}^{\prime}= \begin{cases}(r-1)^{i} / i, & i=1,2, \\ (r-1)^{i} / 2 i, & i \geq 3 .\end{cases}
$$

\section{Contiguity AND ASYmptotic Distributions}

We can easily obtain results on asymptotic distributions under the various (non-uniform) distributions of random (multi-)graphs studied in Section 3.

To begin with, we observe that if $P_{n}$ is the distribution of $G(n, r)$ (or $G^{\prime}(n, r)$; or $\left.G^{*}(n, r)\right)$, then under $P_{n}$ the cycle counts $X_{i n} \stackrel{d}{\rightarrow} Z_{i} \sim \operatorname{Po}\left(\lambda_{i}\right)$, with $Z_{i}$ independent and $\lambda_{i}=\frac{1}{2 i}(r-1)^{i}, i \geq 3$ (or $i \geq 2$; or $i \geq 1$ ). Similarly, if $Q_{n}$ is another of the distributions of multigraphs considered here, then under $Q_{n}$ we have

$$
X_{i n} \stackrel{d}{\rightarrow} Z_{i}^{\prime} \sim \operatorname{Po}\left(\lambda_{i}^{\prime}\right)
$$

with $Z_{i}^{\prime}$ independent, for some $\lambda_{i}^{\prime}$. This follows easily from Proposition 5 , by the argument in Remark 13, in the cases when $d Q_{n} / d P_{n}=Y_{n} / \mathrm{E} Y_{n}$ for a variable $Y_{n}$ satisfying the conclusion of Theorem 1 ; then $\lambda_{i}^{\prime}=\lambda_{i}\left(1+\delta_{i}\right)$. This is, however, going in circles since (4.1) then really is just a reformulation of our assumption A2. We verify A2 and thus (4.1) for several cases in Section 5. Other cases follow similarly; for example, if $Q_{n}$ is the distribution of $G^{*}\left(n, r_{1}\right)+G^{*}\left(n, r_{2}\right), r_{1}+r_{2}=r$, then it is easy to show that (4.1) holds with

$$
\lambda_{i}^{\prime}=\frac{1}{2 i} \operatorname{Tr}\left(\begin{array}{cc}
r_{1}-1 & r_{1} \\
r_{2} & r_{2}-1
\end{array}\right)^{i}=\lambda_{i}\left(1+\left(-\frac{1}{r-1}\right)^{i}\right) .
$$

We write $\lambda_{i}^{\prime}=\lambda_{i}\left(1+\delta_{i}^{\prime}\right)$ and observe that in all examples, $\delta_{i}^{\prime}$ are rather small perturbations. This, in fact, is necessary if $P_{n}$ and $Q_{n}$ are contiguous, since Propositions 7 and 8 then implies $\sum \lambda_{i} \min \left(\delta_{i}^{\prime},\left(\delta_{i}^{\prime}\right)^{2}\right)<\infty$. In all cases we consider, sup $\delta_{i}^{\prime}<\infty$ and we simply have

$$
\sum_{i} \lambda_{i}\left(\delta_{i}^{\prime}\right)^{2}<\infty
$$

Now assume that $Y_{n}$ is a functional of the random regular (multi-)graph, such that under $P_{n}$, for some $\delta_{i} \geq-1$

$$
Y_{n} / \mathrm{E}_{P_{n}} Y_{n} \stackrel{d}{\rightarrow} \prod_{i}\left(1+\delta_{i}\right)^{Z_{i}} e^{-\lambda_{i} \delta_{i}}
$$


jointly with $X_{i n} \stackrel{d}{\rightarrow} Z_{i}$; for example, $Y_{n}$ may be one of the variables studied in Theorems 2-5. Then Proposition 6 applied to $Y_{n} / \mathrm{E}_{P_{n}} Y_{n}$, with $X_{n}=\left(X_{i n}\right)_{i} \in S=\mathbf{R}^{\infty}$ and $f(x)=\prod_{i}\left(1+\delta_{i}\right)^{x_{i}} e^{-\lambda_{i} \delta_{i}}$ (defined to be 0 , for example, when the product diverges), yields that under $Q_{n}$,

$$
Y_{n} / \mathrm{E}_{P_{n}} Y_{n} \stackrel{d}{\longrightarrow} \prod_{i}\left(1+\delta_{i}\right)^{Z_{i}^{\prime}} e^{-\lambda_{i} \delta_{i}}
$$

We thus obtain the asymptotic distribution of $Y_{n}$ under $Q_{n}$, with a suitable normalization, by changing the means of the Poisson variables in (4.3). If we define

$$
a_{n}=\exp \left(\sum_{i} \lambda_{i} \delta_{i} \delta_{i}^{\prime}\right) \mathrm{E}_{P_{n}} Y_{n}
$$

then (4.4) may be rewritten as (under $Q_{n}$ )

$$
Y_{n} / a_{n} \stackrel{d}{\rightarrow} \prod_{i}\left(1+\delta_{i}\right)^{Z_{i}^{\prime}} e^{-\lambda_{i}^{\prime} \delta_{i}}
$$

Here the right hand side has mean 1. If furthermore $a_{n} \sim \mathrm{E}_{Q_{n}} Y_{n}$, we may here replace $a_{n}$ by $\mathrm{E}_{Q_{n}} Y_{n}$ in complete analogy with (4.3). For example, we may do this if both $Y_{n} / \mathrm{E}_{P_{n}} Y_{n}$ and $d Q_{n} / d P_{n}$ are uniformly square integrable for $P_{n}$, because it is easily shown that then $Y_{n} / a_{n}$ is uniformly integrable under $Q_{n}$, and thus $\mathrm{E}_{Q_{n}}\left(Y_{n} / a_{n}\right) \rightarrow 1$.

We can now write down various asymptotic distributions for the variables and models of random regular graphs studied above; we leave the details to the reader.

\section{Proofs of Theorem 1 AND Lemma 1.}

Proof of Theorem 1. We begin by observing that, since $Z_{i} \sim \operatorname{Po}\left(\lambda_{i}\right), \mathrm{E}\left(1+\delta_{i}\right)^{Z_{i}}=$ $e^{\lambda_{i}\left(1+\delta_{i}\right)-\lambda_{i}}=e^{\lambda_{i} \delta_{i}}$ and $\mathrm{E}\left(\left(1+\delta_{i}\right)^{Z_{i}}\right)^{2}=e^{\lambda\left(1+\delta_{i}\right)^{2}-\lambda_{i}}=e^{\lambda\left(2 \delta_{i}+\delta_{i}^{2}\right)}$. Thus, defining

$$
W^{(m)}=\prod_{1}^{m}\left(1+\delta_{i}\right)^{Z_{i}} e^{-\lambda_{i} \delta_{i}}
$$

$W^{(m)}$ is a product of independent variables with mean 1 , and

$$
\mathrm{E}\left(W^{(m)}\right)^{2}=\prod_{1}^{m} e^{\lambda_{i} \delta_{i}^{2}}=\exp \left(\sum_{1}^{m} \lambda_{i} \delta_{i}^{2}\right) \rightarrow \exp \left(\sum_{1}^{\infty} \lambda_{i} \delta_{i}^{2}\right)<\infty \quad \text { as } m \rightarrow \infty .
$$

Hence the sequence $\left(W^{(m)}\right)_{m=1}^{\infty}$ is an $L^{2}$-bounded martingale and thus the limit $W=$ $\lim _{m \rightarrow \infty} W^{(m)}$ exists a.s. and in $L^{2}$, with $\mathrm{E} W^{2}=\exp \left(\sum_{1}^{\infty} \lambda_{i} \delta_{i}^{2}\right)$. We observe for future use that

$$
\mathrm{E}\left|W-W^{(m)}\right|^{2}=\mathrm{E} W^{2}-\mathrm{E}\left(W^{m}\right)^{2}=\exp \left(\sum_{1}^{\infty} \lambda_{i} \delta_{i}^{2}\right)-\exp \left(\sum_{1}^{m} \lambda_{i} \delta_{i}^{2}\right) .
$$


In order to show that $W \neq 0$ a.s., except when $Z_{i}>0$ for some $i$ with $\delta_{i}=-1$, let us break the product defining $W$ into two parts $W_{1}=\prod_{\delta_{i}<-1 / 2}$ and $W_{2}=\prod_{\delta_{i} \geq-1 / 2}$. For $W_{1}$ we observe that although there may conceivably be infinitely many indices in $I_{1}=\left\{i: \delta_{i}<-1 / 2\right\}$, condition A3 implies that $\mathrm{E} \sum_{I_{1}} Z_{i}=\sum_{I_{1}} \lambda_{i} \leq 4 \sum_{I_{1}} \lambda_{i} \delta_{i}^{2}<\infty$, and similarly $\sum_{I_{1}}\left|\lambda_{i} \delta_{i}\right|<\infty$. Hence there are a.s. only finitely many non-zero $Z_{i}, i \in I_{1}$, and $W=e^{-\sum_{I_{1}} \lambda_{i} \delta_{i}} \prod_{I_{1}}\left(1+\delta_{i}\right)^{Z_{i}}$, where the product really is a finite product which is positive unless some factor vanishes, i.e. unless $Z_{i}>0$ for some $i$ with $\delta_{i}=-1$.

For $W_{2}$ we define $\widetilde{\delta}_{i}=-\delta_{i} /\left(1+\delta_{i}\right), i \in I_{2}=\left\{i: \delta_{i} \geq-\frac{1}{2}\right\}$, and note that $\sum_{I_{2}} \lambda_{i} \widetilde{\delta}_{i}^{2}<\infty$ and thus the argument above shows that

$$
\widetilde{W}_{2}=\prod_{I_{2}}\left(1+\widetilde{\delta}_{i}\right)^{Z_{i}} e^{-\lambda_{i} \widetilde{\delta}_{i}}
$$

converges a.s. with $\widetilde{W}_{2}<\infty$. However, since $\left(1+\delta_{i}\right)\left(1+\tilde{\delta}_{i}\right)=1$,

$$
W_{2} \widetilde{W}_{2}=\prod_{I_{2}} e^{-\lambda_{i}\left(\delta_{i}+\widetilde{\delta}_{i}\right)}=\exp \left(-\sum_{I_{2}} \lambda_{i} \delta_{i}^{2} /\left(1+\delta_{i}\right)\right)>0,
$$

so $W_{2}>0$ a.s., which completes the proof that $W>0$ a.s. except when some factor vanishes.

For the rest of the proof we make, without loss of generality, two simplifying assumptions. First we may assume $\mathrm{E} Y_{n}=1$. Secondly, we invoke a theorem by Skorokhod [22] which implies that although the variables $X_{i n}$ and $Y_{n}$ originally may be defined on different probability spaces for different $n$, and $Z_{i}$ on yet another probability space, we may replace them by other variables having the same distributions, such that they become defined on a single probability space and the convergence in A1 holds a.s., i.e. $X_{i n} \rightarrow Z_{i}$ a.s. as $n \rightarrow \infty$ for each $i$.

Fix a large integer $m$ and define the functions

$$
\begin{aligned}
& f_{n}\left(x_{1}, \ldots, x_{m}\right)=\mathrm{E}\left(Y_{n} \mid X_{1 n}=x_{1}, \ldots, X_{m n}=x_{m}\right) \\
& f_{\infty}\left(x_{1}, \ldots, x_{m}\right)=\lim _{n \rightarrow \infty} f_{n}\left(x_{1}, \ldots, x_{m}\right)=\prod_{1}^{m}\left(1+\delta_{i}\right)^{x_{i}} e^{-\lambda_{i} \delta_{i}}
\end{aligned}
$$

where we used assumption A2, and the random variable

$$
Y_{n}^{(m)}=\mathrm{E}\left(Y_{n} \mid X_{1 n}, \ldots, X_{m n}\right)=f_{n}\left(X_{1 n}, \ldots, X_{m n}\right) .
$$

Then $\mathrm{E}\left(Y_{n}^{(m)}\right)^{2}=\sum_{x_{1}, \ldots, x_{m}} \mathrm{P}\left(X_{1 n}=x_{1}, \ldots, X_{m n}=x_{m}\right) f_{n}\left(x_{1}, \ldots, x_{m}\right)^{2}$ and thus, by Fatou's lemma and assumptions A1 and A2,

$$
\begin{aligned}
\liminf _{n \rightarrow \infty} \mathrm{E}\left(Y_{n}^{(m)}\right)^{2} & \geq \sum_{x_{1}, \ldots, x_{m}} \lim _{n \rightarrow \infty} \mathrm{P}\left(X_{1 n}=x_{1}, \ldots, X_{m n}=x_{m}\right) f_{n}\left(x_{1}, \ldots, x_{m}\right)^{2} \\
& =\sum_{x_{1}, \ldots, x_{m}} \mathrm{P}\left(Z_{1}=x_{1}, \ldots, Z_{m}=x_{m}\right) f_{\infty}\left(x_{1}, \ldots, x_{m}\right)^{2} \\
& =\mathrm{E} f_{\infty}\left(Z_{1}, \ldots, Z_{m}\right)^{2}=\mathrm{E}\left(W^{(m)}\right)^{2}=\exp \left(\sum_{1}^{m} \lambda_{i} \delta_{i}^{2}\right) .
\end{aligned}
$$


Consequently, since $Y_{n}^{(m)}$ is a conditional expectation of $Y_{n}$,

$$
\begin{aligned}
\limsup _{n \rightarrow \infty} \mathrm{E}\left|Y_{n}-Y_{n}^{(m)}\right|^{2} & =\underset{n \rightarrow \infty}{\limsup }\left(\mathrm{E} Y_{n}^{2}-\mathrm{E}\left(Y_{n}^{(m)}\right)^{2}\right) \\
& \leq \exp \left(\sum_{1}^{\infty} \lambda_{i} \delta_{i}^{2}\right)-\exp \left(\sum_{1}^{m} \lambda_{i} \delta_{i}^{2}\right) .
\end{aligned}
$$

Furthermore, by our simplifying assumption $X_{i n} \rightarrow Z_{i}$ a.s., we have a.s., for all large $n, X_{i n}=Z_{i}, i \leq m$, and thus

$$
\lim _{n \rightarrow \infty} Y_{n}^{(m)}=\lim _{n \rightarrow \infty} f_{n}\left(Z_{1}, \ldots, Z_{m}\right)=f_{\infty}\left(Z_{1}, \ldots, Z_{m}\right)=W^{(m)} \quad \text { a.s. }
$$

Finally using Chebyshev's inequality, (5.3), (5.2) and (5.1), we obtain for every $\varepsilon>0$,

$$
\begin{aligned}
& \lim \sup \mathrm{P}\left(\left|Y_{n}-W\right|>3 \varepsilon\right) \\
& n \rightarrow \infty \\
& \leq \limsup _{n \rightarrow \infty} \mathrm{P}\left(\left|Y_{n}-Y_{n}^{(m)}\right|>\varepsilon\right)+\limsup _{n \rightarrow \infty} \mathrm{P}\left(\left|Y_{n}^{(m)}-W^{(m)}\right|>\varepsilon\right)+\mathrm{P}\left(\left|W^{(m)}-W\right|>\varepsilon\right) \\
& \leq \varepsilon^{-2} \limsup _{n \rightarrow \infty} \mathrm{E}\left|Y_{n}-Y_{n}^{(m)}\right|^{2}+0+\varepsilon^{-2} \mathrm{E}\left|W-W^{(m)}\right|^{2} \\
& \leq 2 \varepsilon^{-2}\left[\exp \left(\sum_{1}^{\infty} \lambda_{i} \delta_{i}^{2}\right)-\exp \left(\sum_{1}^{m} \lambda_{i} \delta_{i}^{2}\right)\right]
\end{aligned}
$$

We now let $m \rightarrow \infty$, keeping $\varepsilon$ fixed. The right hand side of (5.4) tends to 0 , so the left hand side, which does not depend on $m$, has to vanish for each $\varepsilon>0$, which proves $Y_{n} \stackrel{p}{\rightarrow} W$, and completes the proof of the theorem.

Proof of Lemma 1. Define a new probability measure $Q_{n}$ by $Q_{n}(A)=\mathrm{E}\left(Y_{n} I_{A}\right) / \mathrm{E} Y_{n}$ for every event $A$ in the probability space $\Omega_{n}$ where $X_{i n}$ and $Y_{n}$ are defined. Then the assumption may be written

$$
\mathrm{E}_{Q_{n}}\left(\left(X_{i n}\right)_{j_{1}} \cdots\left(X_{m n}\right)_{j_{n}}\right) \rightarrow \prod_{1}^{m} \mu_{i}^{j_{i}}
$$

and the method of moments yields that under the measures $Q_{n}$,

$$
X_{i n} \stackrel{d}{\rightarrow} \operatorname{Po}\left(\mu_{i}\right)
$$

jointly for all $i$ with independent limits. This and A1 yield, for any $x_{1}, \ldots, x_{m}$,

$$
\begin{aligned}
& \frac{\mathrm{E}\left(Y_{n} \mid X_{1 n}=x_{1}, \ldots, X_{m n}=x_{m}\right)}{\mathrm{E} Y_{n}}=\frac{\mathrm{E}\left(Y_{n} I\left(X_{1 n}=x_{1}, \ldots, X_{m n}=x_{n}\right)\right)}{\mathrm{E} Y_{n} \mathrm{P}\left(X_{1 n}=x_{1}, \ldots, X_{m n}=x_{n}\right)} \\
& \quad=\frac{Q_{n}\left(X_{1 n}=x_{1}, \ldots, X_{m n}=x_{m}\right)}{\mathrm{P}\left(X_{1 n}=X_{1}, \ldots, X_{m n}=x_{m}\right)} \\
& \quad \rightarrow \frac{\prod_{i=1}^{m} \mu_{i}^{x_{i}} e^{-\mu_{i}} / x_{i} !}{\prod_{i=1}^{m} \lambda_{i}^{x_{i}} e^{-\lambda_{i}} / x_{i} !}=\prod_{i=1}^{m}\left(\frac{\mu_{i}}{\lambda_{i}}\right)^{x_{i}} e^{\lambda_{i}-\mu_{i}}
\end{aligned}
$$

which is A2 with $\mu_{i}=\lambda_{i}\left(1+\delta_{i}\right)$. 


\section{Proofs of Theorems 2-9}

We finally show how Theorems 2-9 follow from Theorem 1 . We let $Y_{n}$ be the studied variable and $X_{i n}$ the number of cycles of length $i$ in the random structure. (For the directed case, we use $X_{C n}$ as in Section 1.)

The numbers $\lambda_{i}$ are determined such that A1 holds and then $\delta_{i}$ are given by $\mathrm{A} 2$, see also Remark 5. We will in all cases show A2 by Lemma 1; this usually requires long but rather straight forward combinatorial calculations. It then remains (besides A3) to verify the variance estimate $\mathrm{A} 4$, and that is often the most difficult part of the proof. Fortunately, we can usually just quote the needed result from work by others.

Proof of Theorem 2 and 3. Most steps are the same for the random graphs and multigraphs, so we do both theorems together. We let in the proof $i \geq 1$ in the multigraph case but $i \geq 3$ in the graph case.

A1, with $\lambda_{i}=\frac{1}{2 i}(r-1)^{i}$, is a result by Bollobás [4], [5] and Wormald [23].

For A2 we use Lemma 1 and have to compute factorial moments. This is done in [19] (at least for $G(n, 3)$ ), using generating functions to keep track of the different possibilities for intersections of cycles.

We will here give a slightly different argument, using matrices, which will be easy to adapt to other situations.

We will for simplicity only verify

$$
\frac{\mathrm{E} H_{n}^{*} X_{k n}}{\mathrm{E} H_{n}^{*}} \rightarrow \mu_{k} \quad \text { as } n \rightarrow \infty
$$

the extension to mixed higher factorial moments is routine. We restrict ourselves for the time being to random multigraphs, which (sometimes) are easier to handle than graphs.

Let $V$ be the vertex set of $G^{*}(n, r)$ and $\widetilde{V}=V \times[r]$ the set of half-edges, and let $K(\widetilde{V})$ be the complete graph on $\widetilde{V}$. Let $\widetilde{G}$ denote the random configuration which defines $G^{*}(n, r)$. For any subgraph $A$ of $K(\widetilde{V})$, denote its projection by $A^{0}$; in particular, $\widetilde{G}^{0}=G^{*}(n, r)$.

Let $\widetilde{\mathcal{C}}_{k}$ be the family of all sets of $k$ edges in $K(\widetilde{V})$ that project to a $k$-cycle. Then, using the symmetry,

$$
\frac{\mathrm{E} X_{k n} H_{n}^{*}}{\mathrm{E} H_{n}^{*}}=\frac{\sum_{\widetilde{C} \in \widetilde{\mathcal{C}_{k}}} \sum_{\widetilde{D} \in \widetilde{\mathcal{C}_{n}}} \mathrm{P}(\widetilde{C}, \widetilde{D} \subset \widetilde{G})}{\sum_{\widetilde{D} \in \widetilde{\mathcal{C}_{n}}} \mathrm{P}(\widetilde{D} \subset \widetilde{G})}=\sum_{\widetilde{C} \in \widetilde{\mathcal{C}_{k}}} \sum_{\widetilde{D} \in \widetilde{\mathcal{C}_{n}}} \frac{1}{\left|\widetilde{\mathcal{C}_{n}}\right|} \mathrm{P}(\widetilde{C} \subset \widetilde{G} \mid \widetilde{D} \subset \widetilde{G}) .
$$

We compute this sum as follows. We assume $n>k+1$. We first partition the sum according to the projection $C$ of $\widetilde{C}$, which may be any of the $\frac{1}{2 k}(n)_{k} \sim \frac{1}{2 k} n^{k}$ different $k$ cycles on $V$. (For $k=1$ or 2 , there are slight modifications, which we omit; the final result is the same.) We then further partition the sum according to the graph $B=(\widetilde{C} \cap \widetilde{D})^{0} \subset C$. It is easily seen that the case $B=C$ cannot occur, since it would imply $\widetilde{C} \subset \widetilde{D}$. Now consider one of the remaining $2^{k}-1$ choices of $B ; B$ consists of $j \geq 0$ paths in $C$. Let us traverse $C$ in an arbitrary direction and partition the set of vertices of $C$ into the four sets $V_{00}, V_{01}, V_{10}, V_{11}$ where $V_{00}$ consists of the vertices not adjacent to an edge in $B, V_{01}$ consists of the vertices followed but not preceded by an edge in $B$, and similarly $V_{10}$ and 
$V_{11}$ consist of the vertices preceded and not followed, or followed, respectively, by an edge in $B$. We define $m_{00}=\left|V_{00}\right|, m_{01}=\left|V_{01}\right|, m_{10}=\left|V_{10}\right|$ and $m_{11}=\left|V_{11}\right|$; note that $m_{01}=m_{10}=j$ and that the number of edges in $B$ is $m_{01}+m_{11}$. The Hamilton cycle $D=\widetilde{D}^{0}$ has to contain $B$; these Hamilton cycles in $K_{n}$ may be constructed by selecting a Hamilton cycle on the set of $n-m_{11}-m_{10}$ points obtained by contracting every path in $B$ to a point, and furthermore selecting an orientation for each of these $m_{01}$ paths. Hence there are $2^{m_{01}} \frac{1}{2}\left(n-m_{11}-m_{10}-1\right) ! \sim 2^{m_{01}} n^{-m_{11}-m_{10}}\left|\mathcal{C}_{n}\right|$ choices of $D$, given $C$ and $B$, and thus $\sim 2^{m_{01}} n^{-m_{11}-m_{10}}\left|\widetilde{\mathcal{C}}_{n}\right|$ choices of $\widetilde{D}$.

It remains to choose $\widetilde{C}$. For $C, B$ and $\widetilde{D}$ as above, the edges in $\widetilde{C} \cap \widetilde{D}$ are already determined; to determine the others, we have to make a choice among $r-2$ possibilities at each vertex in $V_{01} \cup V_{10}$ (remembering that 2 of the $r$ original possibilities are used by $\widetilde{D})$, and a choice among $(r-2)(r-3)$ possibilities at each vertex in $V_{00}$. Hence there are $(r-2)^{m_{01}+m_{10}+m_{00}}(r-3)^{m_{00}}$ choices of $\widetilde{C}$. For each of these,

$$
\begin{aligned}
\mathrm{P}(\widetilde{C} \subset \widetilde{G} \mid \widetilde{D} \subset \widetilde{G}) & =\frac{1}{(r n-2 n-1) \cdots\left(r n-2 n-2\left(m_{00}+m_{01}\right)+1\right)} \\
& \sim((r-2) n)^{-m_{00}-m_{01}} .
\end{aligned}
$$

We thus see that the contribution to (6.2) for a given $C$ and $B \neq C$ is

$$
\begin{aligned}
& \sim 2^{m_{01}} n^{-m_{11}-m_{10}}(r-2)^{m_{01}+m_{10}+m_{00}}(r-3)^{m_{00}}((r-2) n)^{-m_{00}-m_{01}} \\
& =2^{m_{01}}(r-2)^{m_{10}}(r-3)^{m_{00}} n^{-k} .
\end{aligned}
$$

By symmetry, all $\frac{1}{2 k}(n)_{k}$ choices of $C$ give the same contribution, so we may fix one of them and obtain (also for $k=1,2$ )

$$
\begin{aligned}
\frac{\mathrm{E} X_{k n} H_{n}^{*}}{\mathrm{E} H_{n}^{*}} & \sim \frac{1}{2 k} \sum_{B \neq C}(r-3)^{m_{00}} 2^{m_{01}}(r-2)^{m_{10}} \\
& =\frac{1}{2 k}\left(\sum_{B \subseteq C}(r-3)^{m_{00}} 2^{m_{01}}(r-2)^{m_{10}}-1\right) .
\end{aligned}
$$

Number the edges in $C e_{1}, \ldots, e_{k}$ (in order). The last sum is over the $2^{k}$ full subgraphs $B \subseteq C$; using the indicators $I\left(e_{i} \in B\right)$ we may rewrite it as a sum over the sequences in $\{0,1\}^{k}$, and it is then seen that the sum equals $\operatorname{Tr}\left(A^{k}\right)$, where $A$ is the matrix

$$
A=\left(\begin{array}{ll}
r-3 & 2 \\
r-2 & 1
\end{array}\right)
$$

We have thus proved (6.1) with

$$
\mu_{k}=\frac{1}{2 k}\left(\operatorname{Tr}\left(A^{k}\right)-1\right)
$$


However, $A$ has the eigenvalues $r-1$ and -1 , and thus $\operatorname{Tr}\left(A^{k}\right)=(r-1)^{k}+(-1)^{k}$. We finally obtain (6.1) and $\mathrm{A} 2^{\prime}$ with

$$
\mu_{k}=\frac{1}{2 k}\left((r-1)^{k}+(-1)^{k}-1\right)=\lambda_{k}\left(1+\frac{(-1)^{k}-1}{(r-1)^{k}}\right)
$$

Lemma 1 now shows that A2 holds with

$$
\delta_{k}=\frac{(-1)^{k}-1}{(r-1)^{k}}= \begin{cases}-2 /(r-1)^{k} & k \text { odd } \\ 0 & k \text { even } .\end{cases}
$$

(It is the fact that $\delta_{k}$ vanishes for even $k$ that makes the number of Hamilton cycles asymptotically independent of the even cycle counts. The calculation above may shed some light on this phenomenon, but it still seems surprising.)

Having verified A2 for the multigraph case, we note that the graph case follows immediately by specializing to $x_{1}=x_{2}=0$, using in particular

$$
\frac{\mathrm{E} H_{n}}{\mathrm{E} H_{n}^{*}}=\frac{\mathrm{E}\left(H_{n}^{*} \mid X_{1 n}=X_{2 n}=0\right)}{\mathrm{E} H_{n}^{*}} \rightarrow e^{-\lambda_{1} \delta_{1}-\lambda_{2} \delta_{2}}=e,
$$

which we obtained in a different way in Section 1.

The values of $\lambda_{i}$ and $\delta_{i}$ yield

$$
\begin{aligned}
\sum_{1}^{\infty} \lambda_{i} \delta_{i}^{2} & =\sum_{i \text { odd }} \frac{2}{i}(r-1)^{-i}=\log \left(1+\frac{1}{r-1}\right)-\log \left(1-\frac{1}{r-1}\right) \\
& =\log \frac{r}{r-2}
\end{aligned}
$$

and thus (for the graph case) $\sum_{3}^{\infty} \lambda_{i} \delta_{i}^{2}=\log \frac{r}{r-2}-\frac{2}{r-1}$. Hence A3 holds, and the variance condition A4 says

$$
\mathrm{E} H_{n}^{2} /\left(\mathrm{E} H_{n}\right)^{2} \rightarrow \frac{r}{r-2} e^{-2 /(r-1)}
$$

in the graph case, and

$$
\mathrm{E} H_{n}^{* 2} /\left(\mathrm{E} H_{n}^{*}\right)^{2} \rightarrow \frac{r}{r-2}
$$

For $r=3,(6.10)$ is proved in [18]. Robinson and Wormald use this case in [19] and they state the result for $r \geq 4$ (when the proof is much more difficult) in [20]. The multigraph case (6.11) has been verified by Frieze, Jerrum and Molloy [9].

This completes the verification of the conditions in Theorem 2 for these two cases.

Remark 14. In fact, (6.11) implies (6.10) by the results of Section 1, see (1.11).

Proof of Theorem 4. We argue as in the preceding proof, but now assume that $\widetilde{D}$ projects to a perfect matching on $V$. Hence $B$ consists of paths of length 1 only, which we account for by a factor $0^{m_{11}}$. (The case $B=C$ is no longer special.) Moreover, no factor $2^{m_{01}}$ 
appears and $\widetilde{D}$ uses only one vertex for each vertex in $V$ so $r-2$ and $r-3$ have to be replaced by $r-1$ and $r-2$. Hence we now take

$$
A=\left(\begin{array}{ll}
r-2 & 1 \\
r-1 & 0
\end{array}\right)
$$

and obtain (6.1) and $\mathrm{A} 2^{\prime}$ with

$$
\mu_{k}=\frac{1}{2 k} \operatorname{Tr}\left(A^{k}\right)=\frac{1}{2 k}\left((r-1)^{k}+(-1)^{k}\right)=\lambda_{k}\left(1+\left(-\frac{1}{r-1}\right)^{k}\right)
$$

since $A$ has the eigenvalues $r-1$ and -1 , and $\lambda_{k}$ is as above.

This yields A2 with

$$
\delta_{k}=\frac{(-1)^{k}}{(r-1)^{k}}, \quad k \geq 1
$$

and

$$
\sum_{1}^{\infty} \lambda_{k} \delta_{k}^{2}=\sum_{1}^{\infty} \frac{1}{2 k}\left(\frac{1}{r-1}\right)^{k}=-\frac{1}{2} \log \left(1-\frac{1}{r-1}\right)=\frac{1}{2} \log \frac{r-1}{r-2}
$$

Hence A4 says

$$
\mathrm{E} M_{n}^{* 2} /\left(\mathrm{E} M_{n}^{*}\right)^{2} \rightarrow \sqrt{\frac{r-1}{r-2}}
$$

for graphs we similarly need

$$
\mathrm{E} M_{n}^{2} /\left(\mathrm{E} M_{n}\right)^{2} \rightarrow \sqrt{\frac{r-1}{r-2}} \exp \left(-\frac{1}{2(r-1)}-\frac{1}{4(r-1)^{2}}\right)
$$

This has been proved by Bollobás and McKay [6] (at least (6.17); (6.16) is similar), which completes the proof.

Proof of Theorem 5. Although we only have been able to complete the proof for $r=3$, we do as much as possible for a general $r \geq 3$ in order to show clearly what is missing in the general case. It suffices to prove the multigraph case; the graph case follows by conditioning as in Section 1.

The probability measure $Q_{n}(A)=\mathrm{E}\left(U_{n}^{*} I_{A}\right) / \mathrm{E} U_{n}^{*}$ is the distribution of the random multigraph obtained as the union of $r$ independent random perfect matchings, $c f$. Section 3. It is easily seen that if $C$ is any of the $\sim n^{i} / 2 i$ cycles of length $i$ in the complete graph, then $C$ appears in this union with probability $\sim N(i, r) n^{-i}$, where $N(i, r)$ is the number of $r$-colourings of the edges in $C$ such that no two adjacent edges have the same colour. Hence we obtain

$$
\mathrm{E}\left(U_{n}^{*} X_{i n}\right) / \mathrm{E} U_{n}^{*}=\mathrm{E}_{Q_{n}}\left(X_{i n}\right) \rightarrow \frac{1}{2 i} N(i, r)
$$

and, more generally by the same argument, $\mathrm{A} 2^{\prime}$ with $\mu_{i}=\frac{1}{2 i} N(i, r)$. Hence A2 holds by Lemma 1. Furthermore, $N(i, r)=\operatorname{Tr} A^{i}$, where $A$ is the $r \times r$-matrix $\left(1-\delta_{i j}\right)_{i, j=1}^{r}$. Since $A$ 
has eigenvalues $r-1$ and -1 , the latter with multiplicity $r-1, N(i, r)=(r-1)^{i}+(r-1)(-1)^{i}$ and

$$
\delta_{i}=\mu_{i} / \lambda_{i}-1=N(i, r) /(r-1)^{i}-1=(-1)^{i}(r-1)^{1-i} .
$$

This gives

$$
\begin{aligned}
\sum_{1}^{\infty} \lambda_{i} \delta_{i}^{2} & =\sum_{1}^{\infty} \frac{1}{2 i}(r-1)^{2-i}=-\frac{(r-1)^{2}}{2} \log \left(1-\frac{1}{r-1}\right) \\
& =\frac{(r-1)^{2}}{2} \log \frac{r-1}{r-2},
\end{aligned}
$$

which verifies $\mathrm{A} 3$ and shows that $\mathrm{A} 4$ is equivalent to

$$
\frac{\mathrm{E}\left(U_{n}^{*}\right)^{2}}{\left(\mathrm{E} U_{n}^{*}\right)^{2}} \rightarrow\left(\frac{r-1}{r-2}\right)^{(r-1)^{2} / 2}
$$

The result now follows by the following moment estimates.

Lemma 3. Let be as above. For any $r \geq 1$, as $n \rightarrow \infty$,

$$
\mathrm{E} U_{n}^{*} \sim 2^{(r-1) / 2}\left(\frac{r !}{r^{r / 2}}\right)^{n}
$$

If $r=3$, then furthermore

$$
\frac{\mathrm{E}\left(U_{n}^{*}\right)^{2}}{\left(\mathrm{E} U_{n}^{*}\right)^{2}} \rightarrow\left(\frac{r-1}{r-2}\right)^{(r-1)^{2} / 2}=4
$$

The proof of Lemma 3 is rather long, and we postpone it to the next section.

Proof of Theorem 6. We argue as for Theorem 4 and obtain, with $A$ given by $(6.12),(6.1)$ and $\mathrm{A} 2{ }^{\prime}$ with

$$
\mu_{k}=\frac{1}{k} \operatorname{Tr} A^{k}=\lambda_{k}\left(1+\left(-\frac{1}{r-1}\right)^{k}\right), \quad k \text { even, }
$$

and thus A2 holds with

$$
\delta_{k}=(r-1)^{-k}, \quad k \text { even. }
$$

Hence

$$
\sum_{i} \lambda_{i} \delta_{i}^{2}=\sum_{j=1}^{\infty} \frac{1}{2 j}(r-1)^{-2 j}=-\frac{1}{2} \log \left(1-(r-1)^{-2}\right)=\frac{1}{2} \log \frac{(r-1)^{2}}{(r(r-2))}
$$

and A4 follows by (1.18), which was proved by Bollobás and McKay [6] (they state the corresponding result for $B(n, r)$, but the argument is the same).

Proof of Theorem 7. We argue as for Theorem 3 in order to estimate $\mathrm{E}\left(H_{n} X_{C n}\right) / \mathrm{E} H_{n}$ for an oriented cycle $C$ of length $k$. 
Let $W$ be the vertex set of $C$, let $d_{c}(v)$ denote the out-degree in $C$ of a vertex $v \in W$, and partition $W$ into $W^{\prime}=\left\{v \in W: d_{C}(v)=1\right\}$ and $W^{\prime \prime}=\left\{v \in W: d_{C}(v)=0\right.$ or 2$\}$. Let also $V_{i j}^{\prime}=W^{\prime} \cap V_{i j}, V_{i j}^{\prime \prime}=W^{\prime \prime} \cap V_{i j}, m_{i j}^{\prime}=\left|V_{i j}^{\prime}\right|$ and $m_{i j}^{\prime \prime}=\left|V_{i j}^{\prime \prime}\right|$. If $m_{11}^{\prime \prime}>0$, i.e. if there is an interior vertex in $B$ with out-degree or in-degree 2 , then no $\widetilde{D}$ exists; we account for that by a factor $0^{m_{11}^{\prime \prime}}$. Moreover, there is no factor $2^{m_{01}}$ and when choosing $\widetilde{C}$ we now have $r-1$ choices at each vertex in $V_{01} \cup V_{10},(r-1)(r-2)$ at each vertex in $V_{00}^{\prime \prime}$ and $(r-1)^{2}$ at each vertex in $V_{00}^{\prime}$; furthermore, $\mathrm{P}(\widetilde{C} \subset \widetilde{G} \mid \widetilde{D} \subset \widetilde{G}) \sim((r-1) n)^{-m_{00}-m_{01}}$.

The total contribution for a given $C$ and $B \neq C$ is thus, cf. (6.3),

$$
\sim n^{-k} 0^{m_{11}^{\prime \prime}}(r-1)^{m_{10}+m_{00}^{\prime}}(r-2)^{m_{00}^{\prime}}
$$

and

$$
\mathrm{E}\left(H_{n} X_{C_{n}}\right) / \mathrm{E} H_{n} \rightarrow \mu_{C}=\frac{1}{\operatorname{aut}(C)} \sum_{B \neq C} 0^{m_{11}^{\prime \prime}}(r-1)^{m_{10}+m_{00}^{\prime}}(r-2)^{m_{00}^{\prime \prime}}
$$

We calculate this sum by adding and subtracting the term with $B=C$, which vanishes except when $C$ is directed; it then equals 1 . Let the vertices of $C$ be $v_{i}, i=1, \ldots, k$, in order (starting from an arbitrary vertex in any direction). The full sum for all $B \subseteq C$ equals $\operatorname{Tr}\left(\prod_{i}^{k} A_{i}\right)$, with $A_{i}=A^{\prime}=\left(\begin{array}{cc}r-1 & 1 \\ r-1 & 1\end{array}\right)$ when $v_{i} \in W^{\prime}$ and $A_{i}=A^{\prime \prime}=\left(\begin{array}{ll}r-2 & 1 \\ r-1 & 0\end{array}\right)$ when $v_{i} \in W^{\prime \prime}$.

These matrices commute (in fact, $A^{\prime}=A^{\prime \prime}+I$ ), so they have common eigenvectors. $A^{\prime}$ has eigenvalues $r$ and 0 , and $A^{\prime \prime}$ has $r-1$ and -1 . Thus

$$
\operatorname{Tr}\left(\prod_{1}^{k} A_{i}\right)=r^{\left|W^{\prime}\right|}(r-1)^{\left|W^{\prime \prime}\right|}+0^{\left|W^{\prime}\right|}(-1)^{\left|W^{\prime \prime}\right|}
$$

and, since $C$ is directed exactly when $W^{\prime \prime}=\emptyset$, we obtain (6.1) and A2' with

$$
\mu_{C}=\frac{1}{\operatorname{aut}(C)}\left(r^{\left|W^{\prime}\right|}(r-1)^{\left|W^{\prime \prime}\right|}+0^{\left|W^{\prime}\right|}(-1)^{\left|W^{\prime \prime}\right|}-0^{\left|W^{\prime \prime}\right|}\right)
$$

Comparing this with (1.19), we see that there are three cases:

(i) If $W^{\prime \prime}=\emptyset$, then $C=C_{k}, \mu_{C_{k}}=\frac{1}{k}\left(r^{k}-1\right)$ and

$$
\delta_{C_{k}}=-1 / r^{k}
$$

(ii) If $W^{\prime}=\emptyset$, then $C=C_{k}^{a}(k$ has to be even $), \mu_{C_{k}}=\frac{1}{k}\left((r-1)^{k}+1\right)$ and

$$
\delta_{C_{k}^{a}}=1 /(r-1)^{k}
$$

(iii) In all other cases, $\mu_{C}=\frac{1}{\operatorname{aut}(C)} r^{\left|W^{\prime}\right|}(r-1)^{\left|W^{\prime \prime}\right|}=\lambda_{C}$ and $\delta_{C}=0$. 
Lemma 1 yields A2 as usual.

$$
\begin{aligned}
\sum \lambda_{C} \delta_{C}^{2} & =\sum_{i=1}^{\infty} \lambda_{C_{i}} \delta_{C_{i}}^{2}+\sum_{j=1}^{\infty} \lambda_{C_{2 j}^{a}} \delta_{C_{2 j}}^{2}=\sum_{1}^{\infty} \frac{1}{i} r^{-i}+\sum_{1}^{\infty} \frac{1}{2 j}(r-1)^{-2 j} \\
& =-\log \frac{r-1}{r}-\frac{1}{2} \log \frac{r(r-2)}{(r-1)^{2}}=\frac{1}{2} \log \frac{r}{r-2}
\end{aligned}
$$

Hence A4 reads

$$
\mathrm{E} H_{n}^{2} /\left(\mathrm{E} H_{n}\right)^{2} \rightarrow \sqrt{\frac{r}{r-2}} .
$$

This can be verified by standard arguments, using the same method as for $G(n, 3)$. (For $r>3$, the calculations for Hamilton cycles in $D(n, r)$ thus are much simpler than for $G(n, r)$.$) We omit the details.$

Proof of Theorem 8. We can either refer to Theorem 6 and the correspondence described in Section 1, or argue as for Theorem 7; the only difference is that now we allow $B=C$, and thus

$$
\mu_{C}=\frac{1}{\operatorname{aut}(C)}\left(r^{\left|W^{\prime}\right|}(r-1)^{\left|W^{\prime \prime}\right|}+0^{\left|W^{\prime}\right|}(-1)^{\left|W^{\prime \prime}\right|}\right),
$$

which gives

$$
\delta_{C_{k}^{a}}=(r-1)^{-k}
$$

and $\delta_{C}=0$ for all other $C$.

Proof of Theorem 9. Consider the configuration model defined by Cooper, Frieze, Molloy and Reed [8]. Take a random configuration, let $Y_{n}$ be the number of perfect matchings and let $X_{i n}$ be the number of cycles of length $i$, see [8] for definitions. It is proved in [8] that $\mathrm{A} 3$ holds with

$$
\lambda_{k}=\frac{((s-1)(r-1))^{k}}{2 k}, \quad k \geq 1 ;
$$

that A2 holds with $\mu_{k}=\lambda_{k}\left(1+(-1)^{k} /(r-1)^{k}\right)$, which by Lemma 1 implies A2 with $\delta_{k}=(-1)^{k} /(r-1)^{k} ;$ and that

$$
\frac{\mathrm{E} Y_{n}^{2}}{\left(\mathrm{E} Y_{n}\right)^{2}} \rightarrow \sqrt{\frac{r-1}{r-s}}
$$

which gives A4 because

$$
\sum_{1}^{\infty} \lambda_{i} \delta_{i}^{2}=\sum_{1}^{\infty} \frac{1}{2 i}\left(\frac{s-1}{r-1}\right)^{i}=-\frac{1}{2} \log \frac{r-s}{r-1} .
$$

The result follows by Theorem 1 and conditioning on $X_{1 n}=0$. 


\section{Proof of Lemma 3}

In order to avoid possible confusion over multiple edges, we work with configurations. We observe that if a multigraph $G^{*}$ is defined by a configuration $\widetilde{G}$, then $U\left(G^{*}\right)$ is the number of $r$-colourings of the edges of $\widetilde{G}$ such that each colour class projects to a perfect matching; we call such colourings proper. We write for simplicity $U=U\left(G^{*}(n, r)\right)=U_{n}^{*}$.

Let $\mathcal{C}$ be the set of all configurations and let us before $V$ be the vertex set of $G^{*}(n, r)$ and $\widetilde{V}$ the set of half-edges. Then

$$
\begin{aligned}
\mathrm{E} U & =\frac{1}{|\mathcal{C}|} \sum_{\widetilde{G} \in \mathcal{C}} \mid\{\text { proper colourings of } \widetilde{G}\} \mid \\
& =\frac{1}{|\mathcal{C}|} \mid\{\text { properly coloured configurations }\} \mid .
\end{aligned}
$$

However, any properly coloured configuration may be constructed by choosing a sequence $M_{1}, \ldots, M_{r}$ of (not necessarily distinct) perfect matchings on $V$, which are to be the projections of the colour classes, and then lifting these to a configuration by assigning endpoints in $\widetilde{V}$ for every edge in the matchings. There are $(n-1) ! !=n ! / 2^{n / 2}(n / 2)$ ! perfect matchings, and every sequence $M_{1}, \ldots, M_{r}$ may be lifted in $(r !)^{n}$ ways, corresponding to selections of one of the $r$ ! possible orderings of the edges at each vertex in $V$. Hence,

$$
\mathrm{E} U=\frac{(n-1) ! !^{r}}{(r n-1) ! !}(r !)^{n} \sim 2^{(r-1) / 2}\left(\frac{r !}{r^{r / 2}}\right)^{n}, \quad r \geq 1,
$$

where we use the asymptotic formula

$$
(n-1) ! !=\frac{n !}{2^{n / 2}(n / 2) !} \sim \sqrt{2}\left(\frac{n}{e}\right)^{n / 2}, \quad n \text { even. }
$$

For $\mathrm{E} U^{2}$ we argue similarly. We define a (proper) double colouring of a configuration to be a pair of proper colourings and obtain

$$
\begin{aligned}
\mathrm{E} U^{2} & =\frac{1}{|\mathcal{C}|} \sum_{\widetilde{G}} \mid\left.\{\text { proper colourings of } \widetilde{G}\}\right|^{2} \\
& =\frac{1}{|\mathcal{C}|} \mid\{\text { doubly coloured configurations }\} \mid
\end{aligned}
$$

For any doubly coloured configuration, we define $r^{2}$ partial matchings $M_{i j}$ on $V$ by taking for every $(i, j) \in\{1, \ldots, r\}^{2}$ the projection of the edges coloured $i$ in the first colouring and $j$ in the second. Let $V_{i j}$ be the vertex set of $M_{i j}$. These sets satisfy the property that if $v \in V$ and $i, j \in\{1, \ldots, r\}$ are given, then $v \in M_{i k}$ for exactly one $k$ and $v \in M_{l j}$ for exactly one $l$. Conversely, any such set of $r^{2}$ matchings comes from exactly $(r !)^{n}$ doubly coloured configurations.

We count the number of such sets of matchings as follows. Given the matchings $M_{i j}$ in sets $V_{i j}$ as above, let, for each permutation $\sigma$ of $\{1, \ldots, r\}, V_{\sigma}=\bigcap_{i} V_{i \sigma(i)}$, i.e. $V_{\sigma}$ is the 
set of all vertices in $V$ that are endpoints of edges in the matchings $M_{i \sigma(i)}, i=1, \ldots, r$. Then $\left(V_{\sigma}\right)_{\sigma \in S_{r}}$ is a partition of $V$. Conversely, given any partition $\left(V_{\sigma}\right)_{\sigma}$ of $V$ such that the sets $V_{i j}=\bigcup_{\sigma: \sigma(i)=j} V_{\sigma}$ have even cardinality, we may choose $M_{i j}$ as arbitrary perfect matchings in $V_{i j}$.

If we let $x_{\sigma}=\left|V_{\sigma}\right| / n$ and

$$
y_{i j}=\left|V_{i j}\right| / n=\sum_{\sigma: \sigma(i)=j} x_{\sigma}
$$

then there are for given $\left(x_{\sigma}\right)_{\sigma \in S_{r}}, n ! / \prod_{\sigma}\left(x_{\sigma} n\right)$ ! ways to choose $\left(V_{\sigma}\right)_{\sigma}$, and for each of these we may choose $M_{i j}$ in $\left(y_{i j} n-1\right)$ !! ways.

Summing up we have,

$$
\mathrm{E} U^{2}=\sum_{x} \frac{1}{(r n-1) ! !}(r !)^{n} \frac{n !}{\prod_{\sigma}\left(x_{\sigma} n\right) !} \prod_{i j}\left(y_{i j} n-1\right) ! !
$$

where we sum over all $x=\left(x_{\sigma}\right)$ such that $x_{\sigma} \geq 0, \sum x_{\sigma}=1, x_{\sigma} n$ are integers and $y_{i j} n$ are even, with $y_{i j}$ defined by (7.2). Note that $\sum_{j} y_{i j}=\sum_{\sigma} x_{\sigma}=1$ for every $i$, and thus $\sum_{i j} y_{i j}=r$.

We now proceed by standard arguments. We use Stirling's formula and obtain, for simplicity ignoring the small modification needed when some $x_{\sigma}=0$,

$$
\begin{aligned}
\mathrm{E} U^{2} & =\sum_{x} 2^{-1 / 2}\left(\frac{r n}{e}\right)^{-r n / 2}(r !)^{n}(2 \pi n)^{(1-r !) / 2} \prod_{\sigma} x_{\sigma}^{-1 / 2-x_{\sigma} n} 2^{r^{2} / 2} \prod_{i j}\left(\frac{y_{i j} n}{e}\right)^{y_{i j} n / 2} \\
& =\sum_{x} 2^{\left(r^{2}-1\right) / 2}(2 \pi n)^{(1-r !) / 2} \prod_{\sigma} x_{\sigma}^{-1 / 2}\left(\frac{r !}{r^{r / 2}} e^{f(x)}\right)^{n}\left(1+O\left(\max _{\sigma}\left(x_{\sigma} n\right)^{-1}\right)\right)
\end{aligned}
$$

where

$$
f(x)=-\sum_{\sigma} x_{\sigma} \log x_{\sigma}+\sum_{i, j} \frac{1}{2} y_{i j} \log y_{i j} .
$$

As is well-known, the asymptotic behaviour of such a sum depends only on the behaviour of $f$ near its maxima.

Define $x^{*}=\left(x_{\sigma}^{*}\right)$, with every $x_{\sigma}^{*}=1 / r$ !. The corresponding $y_{i j}$ are all $1 / r$, and thus

$$
f\left(x^{*}\right)=-\log \frac{1}{r !}+\frac{r}{2} \log \frac{1}{r}=\log r !-\frac{r}{2} \log r .
$$

Lemma 4. If $r=3$, then $f(x)$ has on the simplex $\left\{x: x_{\sigma} \geq 0, \sum x_{\sigma}=1\right\}$ a unique global maximum at $x=x^{*}$.

We postpone the proof of this lemma. 
We conjecture that the lemma is true also for $r>3$, but we have not been able to verify it; this is the reason Lemma 4 and Theorem 5 are restricted to $r=3$. (If $r=2, f$ vanishes identically and the lemma fails.)

For any $r$ such that the lemma is true (in particular for $r=3$ ), the sum in (7.4) is dominated by terms with $x-x^{*}$ small, say $\left|x-x^{*}\right|<n^{-3 / 8}$. For such $x$ we use the Taylor expansion

$$
f(x)=f\left(x^{*}\right)-\frac{1}{2}\left(x-x^{*}\right)^{t} B\left(x-x^{*}\right)+O\left(\left|x-x^{*}\right|^{3}\right)
$$

where $-B$ is the matrix $\left(\frac{\partial^{2} f}{\partial x_{\sigma} \partial x_{\tau}}\left(x^{*}\right)\right)$ of second derivatives of $f$ at $x^{*}$. We will shortly see that $B$ is non-singular, and since $x^{*}$ is a maximum point, $B$ has to be positive definite on the hyperplane $\sum x_{\sigma}=0$.

We now approximate the sum in (7.4) by an $r !-1$ dimensional integral. We fix an arbitrary permutation $\sigma_{0}$ and write $\hat{d} x$ for $\prod_{\sigma \neq \sigma_{0}} d x_{\sigma}$. Given that $\sum_{\sigma} x_{\sigma} n=n$, an even integer, the conditions that $y_{i j} n$ be even for all $i, j \in\{1, \ldots, r-1\}$ yield $(r-1)^{2}$ linearly independent conditions (over $G F(2)$ ) that imply that also the remaining $y_{i j} n$ are even. Hence only $2^{-(r-1)^{2}}$ of the possible values of $\left(x_{\sigma} n \bmod 2\right)_{\sigma}$ are allowed. We thus obtain, $\mathrm{E} U^{2} \sim$

$$
\int_{\sum x_{\sigma}=1} 2^{\left(r^{2}-1\right) / 2}(2 \pi n)^{(1-r !) / 2}(r !)^{r ! / 2}\left(\frac{r !}{r^{r / 2}} e^{f\left(x^{*}\right)}\right)^{n} e^{-\frac{1}{2} n\left(x-x^{*}\right) B\left(x-x^{*}\right)} 2^{-(r-1)^{2}} n^{r !-1} \hat{d} x .
$$

This Gaussian integral may be evaluated by several methods. For reference we state one method as a lemma, whose proof we omit.

Lemma 5. Suppose that $B$ is a non-singular symmetric $N \times N$ matrix which is positive definite on the hyperplane $\sum x_{i}=0$ and such that $(1, \ldots, 1)$ is an eigenvector with eigenvalue $\lambda_{1}$. Then

$$
\int_{\sum x_{i}=0} e^{-\frac{1}{2} x^{t} B x} \hat{d} x=\frac{1}{\sqrt{N}}(2 \pi)^{(N-1) / 2}\left(\frac{\lambda_{1}}{|B|}\right)^{1 / 2} .
$$

The second derivatives of $f$ are given by

$$
\frac{\partial^{2} f}{\partial x_{\sigma} \partial x_{\tau}}=-\frac{1}{x_{\sigma}} \delta_{\sigma \tau}+\frac{1}{2} \sum_{i: \sigma(i)=\tau(i)} \frac{1}{y_{i j}}
$$

and thus

$$
B=\left(r ! \delta_{\sigma \tau}-\frac{r}{2}|\{i: \sigma(i)=\tau(i)\}|\right)_{\sigma \tau} .
$$

It follows that $(1, \ldots, 1)$ is an eigenvector of $B$ with eigenvalue $\lambda_{1}=\left(1-\frac{r}{2}\right) r$ !. Moreover, the determinant is given by

$$
|B|=\lambda_{1}\left(\frac{r-2}{2(r-1)} r !\right)^{(r-1)^{2}} r !^{r !-1-(r-1)^{2}}
$$


by an argument using group representation theory, which shows that $B$ has besides $\lambda_{1}$ the eigenvalues $\frac{r-2}{2(r-1)} r$ ! with multiplicity $(r-1)^{2}$ and $r$ ! with multiplicity $r !-(r-1)^{2}-1$. We omit the details since the case $r=3$ also can be easily verified by direct evaluation of the determinant. We thus obtain, for $r$ such that Lemma 4 is valid,

$$
\begin{aligned}
\mathrm{E} U^{2} & \sim 2^{\left(r^{2}-1\right) / 2} r !^{r ! / 2}\left(\frac{r !}{r^{r / 2}}\right)^{2 n} 2^{-(r-1)^{2}} r !^{-1 / 2}\left(\frac{\lambda_{1}}{|B|}\right)^{1 / 2} \\
& =2^{\left(r^{2}-1\right) / 2-(r-1)^{2}}\left(\frac{2(r-1)}{r-2}\right)^{(r-1)^{2} / 2}\left(\frac{r !}{r^{r / 2}}\right)^{2 n} \\
& =2^{r-1}\left(\frac{r-1}{r-2}\right)^{(r-1)^{2} / 2}\left(\frac{r !}{r^{r / 2}}\right)^{2 n}
\end{aligned}
$$

and

$$
\mathrm{E} U^{2} /(\mathrm{E} U)^{2} \sim\left(\frac{r-1}{r-2}\right)^{(r-1)^{2} / 2} \text {. }
$$

Proof of Lemma 4. Let us first assume that $x$ is an extreme point with every $x_{\sigma}>0$. By the Lagrange multiplier method, there exists $\lambda$ such that for all $\sigma$

$$
\lambda=\frac{\partial f}{\partial x_{\sigma}}=-\log x_{\sigma}-1+\frac{1}{2} \sum_{i}\left(\log y_{i \sigma(i)}+1\right)
$$

and thus for $a=\exp (2 \lambda+2-r)>0$,

$$
a x_{\sigma}^{2}=\prod_{i} y_{i \sigma(i)}
$$

We now employ the special properties of $r=3$. We relabel the $x_{\sigma}$ as $x_{1}, x_{2}, x_{3}, x_{1}^{\prime}, x_{2}^{\prime}, x_{3}^{\prime}$, with the $x_{i}$ corresponding to even permutations $\sigma$, and $x_{i}^{\prime}$ to odd ones. It is easily seen that each $y_{i j}$ is a sum $x_{\sigma_{1}}+x_{\sigma_{2}}$ where one of the permutations is even and the other odd, i.e. $y_{i j}=x_{k}+x_{l}^{\prime}$ for some $k$ and $l$.

Hence, if $\sigma$ is even, $y_{i \sigma(i)}=x_{\sigma}+x_{\tau}$, where $\tau$ runs through the three odd permutations for $i=1,2,3$. Hence (7.5) yields

$$
a x_{i}^{2}=\prod_{j}\left(x_{i}+x_{j}^{\prime}\right)
$$

and similarly

$$
a x_{j}^{\prime 2}=\prod_{i}\left(x_{i}+x_{j}^{\prime}\right)
$$

Consider now the polynomial

$$
p(x)=\prod_{j}\left(x+x_{j}^{\prime}\right)-\prod_{i}\left(x-x_{i}\right)-a x^{2}
$$


which has degree at most 2 and is not identically zero because $p(0)=\prod_{j} x_{j}^{\prime}+\prod_{i} x_{i}>0$. By (7.6) and (7.7), $x_{1}, x_{2}, x_{3},-x_{1}^{\prime},-x_{2}^{\prime},-x_{3}^{\prime}$ are all roots, and this is possible only if $x_{1}=x_{2}=x_{3}$ and $x_{1}^{\prime}=x_{2}^{\prime}=x_{3}^{\prime}$. (7.6) and (7.7) now yield $a x_{i}^{2}=\left(x_{1}+x_{1}^{\prime}\right)^{3}=a x_{j}^{\prime 2}$, and thus also $x_{i}=x_{j}^{\prime}$; hence $x=x^{*}$.

This shows that $x^{*}$ is the only extreme point in the interior of the simplex.

Now suppose that $x$ is a maximum point at the boundary. If $x_{\sigma}=0$ but $y_{i \sigma(i)}>0$ for all $i$, then increasing $x_{\sigma}$ to $\varepsilon>0$, at the expense of some positive $x_{\tau}$, with increase $f(x)$ by $-\varepsilon \log \varepsilon+O(\varepsilon)>0$ if $\varepsilon$ is small; similarly, if only one $y_{i \sigma(i)}=0, f(x)$ will increase by $-\frac{1}{2} \varepsilon \log \varepsilon+O(\varepsilon)>0$. It follows that if $x$ is a maximum point, then $y_{i \sigma(i)}=0$ for at least two $i$ whenever $x_{\sigma}=0$. This implies easily that $x_{\sigma} \neq 0$ for at most one odd and one even permutation, $\tau$ and $\nu$, say, but then of the nine $y_{i j}$, four equal 0 , two equal $x_{\tau}$ and two $x_{\nu}$, and one equal $x_{\tau}+x_{\nu}=1$. Hence $f(x)=0<f\left(x^{*}\right)$, which completes the proof that the global maximum is attained at $x^{*}$, and only there.

\section{REFERENCES}

1. A. D. Barbour, S. Janson, M. Karoński and A. Ruciński, Small cliques in random graphs, Random Struct. Alg. 1 (1990), 403-434.

2. P. Billingsley, Convergence of Probability Measures, Wiley, New York, 1968.

3. E.A. Bender and E.R. Canfield, The asymptotic number of labeled graphs with given degree sequences, J. Combin. Th. A 24 (1978), 296-307.

4. B. Bollobás, A probabilistic proof of an asymptotic formula for the number of labelled regular graphs, Europ. J. Combinatorics 1 (1980), 311-316.

5. B. Bollobás, Random Graphs, Academic Press, London, 1985.

6. B. Bollobás and B.D. McKay, The number of matchings in random regular graphs and bipartite graphs, J. Combin. Th. B 41 (1986), 80-91.

7. C. Cooper, A. Frieze and M. Molloy, Hamilton cycles in random regular digraphs (to appear).

8. C. Cooper, A. Frieze, M. Molloy and B. Reed, Perfect matchings in random $r$-regular, $s$-uniform hypergraphs (to appear).

9. A.M. Frieze, M.R. Jerrum and M. Molloy, Constructing and counting Hamilton cycles in random regular graphs, in preparation.

10. A. Frieze and S. Suen, Counting Hamilton cycles in random directed graphs, Random Struct. Alg. 3 (1992), 235-242.

11. S. Janson, Orthogonal decompositions and functional limit theorems for random graph statistics, Memoirs Amer. Math. Soc. (to appear).

12. S. Janson, The numbers of spanning trees, Hamilton cycles and perfect matchings in a random graph, Combinatorics, Probability and Computing (to appear).

13. L. Le Cam, Locally asymptotically normal families of distributions, Univ. of California Publ. in Statistics 3 (1960), 37-98.

14. L. Le Cam, Théorie asymptotique de la décision statistique, Les Presses de l'Université de Montréal, Montreal, 1969.

15. L. Le Cam, Asymptotic methods in statistical decision theory, Springer-Verlag, New York, 1986.

16. M. Loève, Probability theory, 4th ed., Springer-Verlag, New York, 1977.

17. M. Molloy, H. Robalewska-Szarłat, R.W. Robinson and N.C. Wormald, 1-factorisations of random regular graphs, in preparation.

18. R.W. Robinson and N.C. Wormald, Existence of long cycles in random cubic graphs, Enumeration and Design (D.M. Jackson and S.A. Vanstone, eds.), Academic Press, Toronto, 1984, pp. 251-270.

19. R.W. Robinson and N.C. Wormald, Almost all cubic graphs are hamiltonian, Random Struct. Alg. 3 (1992), 117-125.

20. R.W. Robinson and N.C. Wormald, Almost all regular graphs are hamiltonian, Random Struct. Alg. (to appear). 
21. G. Roussas, Contiguity of probability measures: some applications in statistics, Cambridge Univ.

22. A.V. Skorokho

289-319 (Russian); English trans for stochastic processes, Teor. Veroyatnost. i Primenen. 1 (1956),

23. N.C. Wormald, The asymptoticl., Theor. Probab. Appl. 1 (1956), 261-290.

$B 31$ (1981), 168-182.

Svante Janson, Department of Mathematics, Uppsala University, PO Box 480 , S-751 06 UPPSALA, SWEDEN

E-mail address: svante.janson@math.uu.se 
1108 Lawrence Markus, A brief history of control

Richard A. Brualdi, Keith L. Chavey \& Bryan L. Shader, Bipartite graphs and inverse sign patterns of strong sign-nonsingular matrices

A. Kersch, W. Morokoff \& A. Schuster, Radiative heat transfer with quasi-monte carlo methods

Jianhua Zhang, A free boundary problem arising from swelling-controlled release processes

Walter Littman \& Stephen Taylor, Local smoothing and energy decay for a semi-infinite beam pinned at several points and applications to boundary control

Srdjan Stojanovic \& Thomas Svobodny, A free boundary problem for the Stokes equation via nonsmooth analysis

Bronislaw Jakubczyk, Filtered differential algebras are complete invariants of static feedback

Boris Mordukhovich, Discrete approximations and refined Euler-Lagrange conditions for nonconvex differential inclusions

Bei Hu \& Hong-Ming Yin, The profile near blowup time for solution of the heat equation with a nonlinear boundary condition

Jin Ma \& Jiongmin Yong, Solvability of forward-backward SDEs and the nodal set of Hamilton-Jacobi-Bellman Equations

Chaocheng Huang \& Jiongmin Yong, Coupled parabolic and hyperbolic equations modeling age-dependent epidemic dynamics with nonlinear diffusion

Jiongmin Yong, Necessary conditions for minimax control problems of second order elliptic partial differential equations

Eitan Altman \& Nahum Shimkin, Worst-case and Nash routing policies in parallel queues with uncertain service allocations

Nahum Shimkin \& Adam Shwartz, Asymptotically efficient adaptive strategies in repeated games, part II: Asymptotic optimality

M.E. Bradley, Well-posedness and regularity results for a dynamic Von Kármán plate

1124

Zhangxin Chen, Finite element analysis of the 1D full drift diffusion semiconductor model

Gang Bao \& David C. Dobson, Diffractive optics in nonlinear media with periodic structure

Steven Cox \& Enrique Zuazua, The rate at which energy decays in a damped string

1126

1127

1128

Anthony W. Leung, Optimal control for nonlinear systems of partial differential equations related to ecology

H.J. Sussmann, A continuation method for nonholonomic path-finding problems

Yung-Jen Guo \& Walter Littman, The null boundary controllability for semilinear heat equations

1129

Q. Zhang \& G. Yin, Turnpike sets in stochastic manufacturing systems with finite time horizon

1130

1131

1132

1133

1134

1135

1136

1137

1138

1139

I. Györi, F. Hartung \& J. Turi, Approximation of functional differential equations with time- and state-dependent delays by equations with piecewise constant arguments

I. Györi, F. Hartung \& J. Turi, Stability in delay equations with perturbed time lags

F. Hartung \& J. Turi, On the asymptotic behavior of the solutions of a state-dependent delay equation

Pierre-Alain Gremaud, Numerical optimization and quasiconvexity

Jie Tai Yu, Resultants and inversion formula for $N$ polynomials in $N$ variables

Avner Friedman \& J.L. Velázquez, The analysis of coating flows in a strip

Eduardo D. Sontag, Control of systems without drift via generic loops

Yuan Wang \& Eduardo D. Sontag, Orders of input/output differential equations and state space dimensions

Scott W. Hansen, Boundary control of a one-dimensional, linear, thermoelastic rod

Robert Lipton \& Bogdan Vernescu, Homogenization of two phase emulsions with surface tension effects

Scott Hansen \& Enrique Zuazua, Exact controllability and stabilization of a vibrating string with an interior point mass

1141 Bei Hu \& Jiongmin Yong, Pontryagin Maximum principle for semilinear and quasilinear parabolic equations with pointwise state constraints

1142 Mark H.A. Davis, A deterministic approach to optimal stopping with application to a prophet inequality

M.H.A. Davis \& M. Zervos, A problem of singular stochastic control with discretionary stopping

Bernardo Cockburn \& Pierre-Alain Gremaud, An error estimate for finite element methods for scalar conservation laws

David C. Dobson \& Fadil Santosa, An image enhancement technique for electrical impedance tomography

Jin Ma, Philip Protter, \& Jiongmin Yong, Solving forward-backward stochastic differential equations explicitly - a four step scheme

Yong Liu, The equilibrium plasma subject to skin effect

Ulrich Hornung, Models for flow and transport through porous media derived by homogenization

Avner Friedman, Chaocheng Huang, \& Jiongmin Yong, Effective permeability of the boundary of a domain Gang Bao, A uniqueness theorem for an inverse problem in periodic diffractive optics

Angelo Favini, Mary Ann Horn,\& Irena Lasiecka, Global existence and uniqueness of regular solutions to the dynamic von Kármán system with nonlinear boundary dissipation 
E.G. Kalnins \& Willard Miller, Jr., Models of $q$-algebra representations: $q$-integral transforms and "addition theorems"

1153 E.G. Kalnins, V.B. Kuznetsov \& Willard Miller, Jr., Quadrics on complex Riemannian spaces of constant curvature, separation of variables and the Gaudin magnet

A. Kersch, W. Morokoff \& Chr. Werner, Selfconsistent simulation of sputtering with the DSMC method Bing-Yu Zhang, A remark on the Cauchy problem for the Korteweg-de Vries equation on a periodic domain

Gang Bao, Finite element approximation of time harmonic waves in periodic structures

Tao Lin \& Hong Wang, Recovering the gradients of the solutions of second-order hyperbolic equations by interpolating the finite element solutions

Zhangxin Chen, $L^{p}$-posteriori error analysis of mixed methods for linear and quasilinear elliptic problems

Todd Arbogast \& Zhangxin Chen, Homogenization of compositional flow in fractured porous media

L. Qiu, B. Bernhardsson, A. Rantzer, E.J. Davison, P.M. Young \& J.C. Doyle, A formula for computation of the real stability radius

Maria Inés Troparevsky, Adaptive control of linear discrete time systems with external disturbances under inaccurate modelling: A case study

1162 Petr Klouček \& Franz S. Rys, Stability of the fractional step $\Theta$-scheme for the nonstationary Navier-Stokes equations

1163 Eduardo Casas, Luis A. Fernández \& Jiongmin Yong, Optimal control of quasilinear parabolic equations

Darrell Duffie, Jin Ma \& Jiongmin Yong, Black's consol rate conjecture

D.G. Aronson \& J.L. Vazquez, Anomalous exponents in nonlinear diffusion

Ruben D. Spies, Local existence and regularity of solutions for a mathematical model of thermomechanical phase transitions in shape memory materials with Landau-Ginzburg free energy

Pu Sun, On circular pipe Poiseuille flow instabilities

Angelo Favini, Mary Ann Horn, Irena Lasiecka \& Daniel Tataru, Global existence, uniqueness and regularity of solutions to a Von Kármán system with nonlinear boundary dissipation

A. Dontchev, Tz. Donchev \& I. Slavov, On the upper semicontinuity of the set of solutions of differential

inclusions with a small parameter in the derivative
Jin Ma \& Jiongmin Yong, Regular-singular stochastic controls for higher dimensional diffusions — dynamic programming approach

1171 Alex Solomonoff, Bayes finite difference schemes

1172 Todd Arbogast \& Zhangxin Chen, On the implementation of mixed methods as nonconforming methods for second order elliptic problems

1173 Zhangxin Chen \& Bernardo Cockburn, Convergence of a finite element method for the drift-diffusion semiconductor device equations: The multidimensional case

Boris Mordukhovich, Optimization and finite difference approximations of nonconvex differential inclusions with free time

Avner Friedman, David S. Ross, and Jianhua Zhang, A Stefan problem for reaction-diffusion system

Alex Solomonoff, Fast algorithms for micromagnetic computations

Nikan B. Firoozye, Homogenization on lattices: Small parameter limits, $H$-measures, and discrete Wigner measures

G. Yin, Adaptive filtering with averaging

Wlodzimierz Byrc and Amir Dembo, Large deviations for quadratic functionals of Gaussian processes

Ilja Schmelzer, 3D anisotropic grid generation with intersection-based geometry interface

Alex Solomonoff, Application of multiple methods to two matrix eigenproblems

A.M. Latypov, Numerical solution of steady euler equations in streamline-aligned orthogonal coordinates

Bei Hu \& Hong-Ming Yin, Semilinear parabolic equations with prescribed energy

Bei Hu \& Jianhua Zhang, Global existence for a class of Non-Fickian polymer-penetrant systems

Rongze Zhao \& Thomas A. Posbergh, Robust stabilization of a uniformly rotating rigid body

Mary Ann Horn \& Irena Lasiecka, Uniform decay of weak solutions to a von Kármán plate with nonlinear boundary dissipation

Mary Ann Horn, Irena Lasiecka \& Daniel Tataru, Well-posedness and uniform decay rates for weak solutions to a von Kármán system with nonlinear dissipative boundary conditions

Mary Ann Horn, Nonlinear boundary stabilization of a von Kármán plate via bending moments only

Frank H. Shaw \& Charles J. Geyer, Constrained covariance component models

Tomasz Luczaka, A greedy algorithm estimating the height of random trees

Timo Seppäläinen, Maximum entropy principles for disordered spins

Yuandan Lin, Eduardo D. Sontag \& Yuan Wang, Recent results on Lyapunov-theoretic techniques for nonlinear stability

Svante Janson, Random regular graphs: Asymptotic distributions and contiguity

Rachid Ababou, Random porous media flow on large 3-D grids: Numerics, performance, \& application to homogenization 